\title{
The dragonflies and damselflies (Odonata) of Angola
}

\author{
Jens Kipping ${ }^{1,2}$, Viola Clausnitzer ${ }^{3}$, Sara R. F. Fernandes Elizalde ${ }^{4}$, \\ Klaas-Douwe B. Dijkstra ${ }^{5,6}$
}

I BioCart Ökologische Gutachten, Albrecht-Dürer-Weg 8, D-04425 Taucha/Leipzig, Germany 2 Anhalt University of Applied Sciences, Strenzfelder Allee 28, 06406 Bernburg, Germany 3 Senckenberg Museum for Natural History, Görlitz, Am Museum 1, 02826 Görlitz, Germany 4 SASSCAL - BID GBIF, Instituto de Investigação Agronómica, Chianga, Huambo, Angola 5 Department of Conservation Ecology and Entomology, University of Stellenbosch, South Africa 6 Naturalis Biodiversity Center, P.O. Box 9517, 2300 RA Leiden, The Netherlands

Corresponding author: Jens Kipping (biocartkipping@web.de)

Academic editor: Pavel Stoev | Received 9 December 2016 | Accepted 20 March 2017 | Published 12 April 2017

http://zoobank.org/FEDF8E20-0A7E-49BF-B883-9558BDF1037A

Citation: Kipping J, Clausnitzer V, Elizalde SRF, Dijkstra K-DB (2017) The dragonflies and damselflies (Odonata) of Angola. African Invertebrates 58(1): 65-91. https://doi.org/10.3897/AfrInvertebr.58.11382

\begin{abstract}
Prior to 2012, only 158 species of Odonata were known from Angola. Surveys in 2012 and 2013 added 76 species and two further additions in 2016 brought the national total to 236 species. We provide a revised checklist with taxonomic notes and discuss the history of research, the biogeography of the fauna, and the potential for further discoveries. The national total is likely to be above 300 species. This would make Angola one of the richest countries for Odonata in Africa. The endemic species formerly classified in Chlorocypha are transferred to Platycypha.
\end{abstract}

\section{Resumo}

Até 2012, apenas eram conhecidas 158 espécies de Odonata em Angola. Levantamentos feitos em 2012 e 2013 acrescentaram 76 espécies que, em conjunto com dois novos registos de 2016, elevaram a lista nacional a 236 espécies. Apresentamos uma lista de referência revista, com notas taxonómicas e discussão histórica da investigaçáo, a biogeografia da fauna e o potencial de novas descobertas. É provável que o número total de espécies no país seja superior a 300. Se assim for, Angola será um dos países africanos com maior riqueza em Odonata. As espécies endémicas anteriormente classificadas como Chlorocypha foram transferidas para Platycypha. 


\section{Keywords}

Africa, biogeography, checklist, diversity, conservation

\section{Palavras-chave}

África, biogeografia, checklist, diversidade, conservação

\section{Introduction}

Covering nearly 1,250,000 $\mathrm{km}^{2}$, Angola is the largest African country not dominated by the Sahara after the Democratic Republic of Congo. It has an accordingly high diversity of habitats, ranging from Namib Desert in the southwest through grassland, woodlands and montane forest to Congolian lowland rainforest in the northeast. Moreover, a great highland of up to 2,620 m asl (above sea level) is contained completely within Angola's borders, leading to substantial endemism. Therefore, the country is likely to be one of the richest in species in Africa. However, Angola's biodiversity is very poorly known, with comparatively limited research before independence in 1975 halting altogether in the three decades of unrest that followed. Research coverage is also limited for Odonata (Fig. 1), with much of the north and east never surveyed at all (Clausnitzer et al. 2012). The potentially very species-rich highland catchments of the Congo, Cuanza, Cubango (Okavango) and Zambezi Rivers are almost unknown and may hold many undescribed species. The whole Angolan part of the extensively marshy Cuando River and almost the entire Cuito river system are also unsurveyed.

\section{History of Odonata research in Angola}

Research began in July 1928, when the Swiss zoologist Albert Monard embarked on the first of his two expeditions to Angola, which lasted until February 1929. Monard was a curator at the Natural Museum of La-Chaux-de-Fonds in Switzerland with a broad interest in nature who mainly collected vertebrates and plants. Ris (1931) identified 27 and described four species from Monard's first expedition.

With the death of Friedrich Ris, Monard submitted the Odonata from his second expedition (April 1932 to October 1933) to Cynthia Longfield at the British Museum (now the Natural History Museum) in London, who had published several records obtained by Karl Jordan from Mt Moco in 1934 (Longfield 1936). Longfield (1947) identified 77 species from Monard's new material and described 13 new species and two new genera. She also dealt with the Odonata held at the Dundo Museum in northern Angola, first revising the genus Orthetrum based on the long series available (Longfield 1955) and later listing 61 species from the collection, including three new ones (Longfield 1959).

Pinhey (1961a, b) described five new species of Gomphidae from northern Angola received from A. de Barros Machado of the Dundo Museum. While Longfield (1959) 


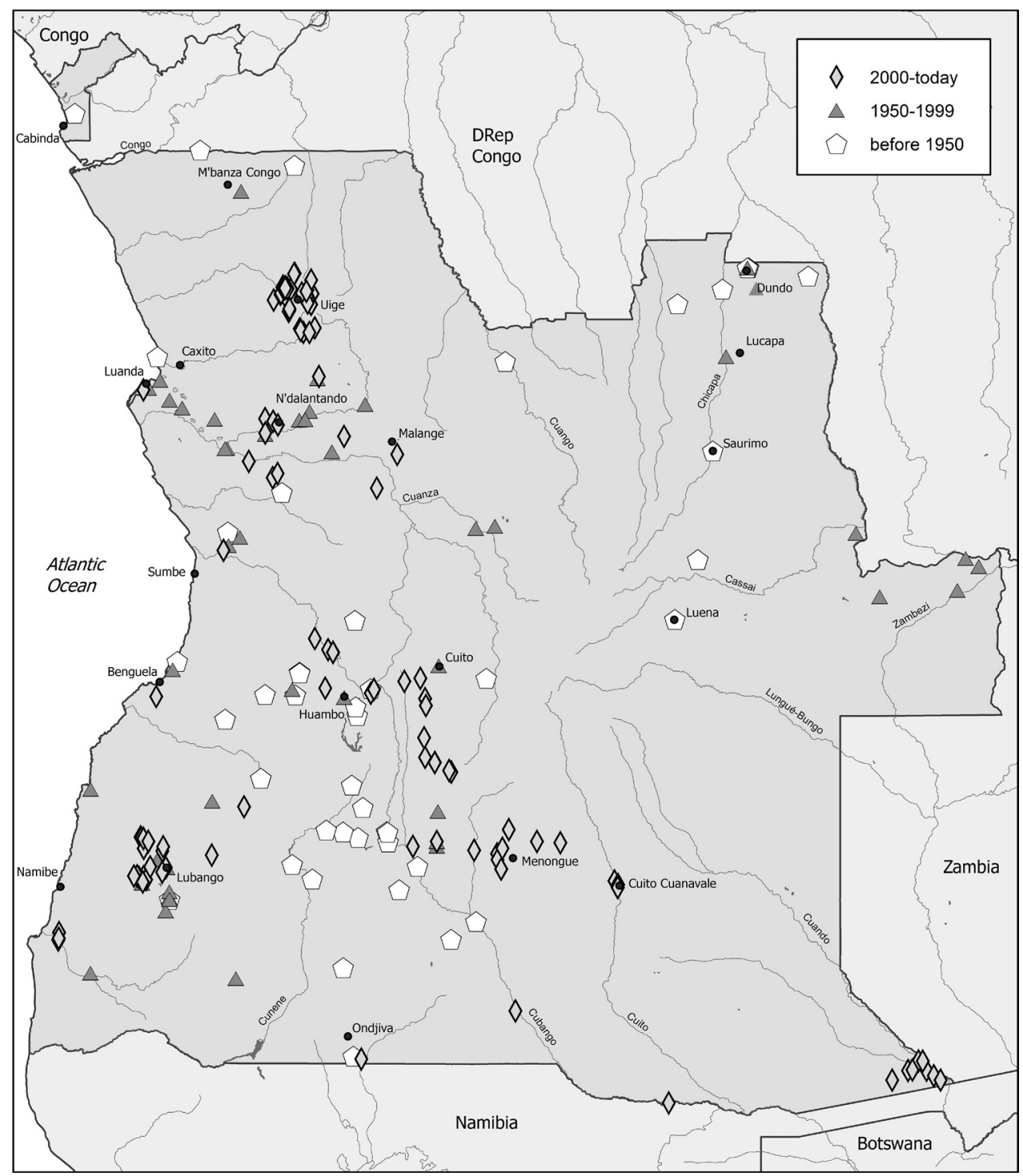

Figure I. Records of Odonata from Angola before 1950, before 2000 and up to 2016.

stated that the Dundo collection "shows the usual scarcity of the genera Gomphidae", Pinhey (1961a) noted it "was particularly notable for the number of Gomphids." Possibly Machado split the material between the two authors. It is uncertain whether the material was collected in Dundo or only held there, as most records lack details on collector, date and precise locality. However, Pinhey (1961b) did detail collecting localities around Dundo, suggesting that all material came from this part of Lunda Norte Province. The collector was probably Machado himself. No-one has worked on this collection since and its state is thus unknown. 
Elliot Pinhey was curator at the National Museum of Zimbabwe from 1955 until 1975 and while he collected intensively in adjacent countries, only visited Angola twice (Vick et al. 2001). In April and May 1963 Pinhey participated in an expedition to north-western Zambia, also visiting an area east of Caianda and the Lutchigena River in Angola directly adjacent to the Ikelenge Pedicle of Zambia, where he recorded 26 species (Pinhey 1964, 1974, 1984). His second excursion into Angola went to an area between Luanda and the Duque de Bragança Falls on the Lucala River (now known as Kalandula Falls) in October 1964 with records of 32 species (Pinhey 1965).

Pinhey further treated the material of three collectors, describing a species in honour of each of them. Edward S. Ross of the California Academy of Sciences collected between Cuchi and Dondo in 1957 and 1958 (Pinhey 1966), the famed expert of mammal behaviour Richard Estes in central Angola in 1970 (Pinhey 1971a), and Ivan Bampton around the Serra da Chela and Tundavala in 1973 (Pinhey 1975). In the 1975 paper he also repeated records from Pinhey $(1964,1965)$ and Longfield (1947), and provided a gazetteer, causing confusion about the precise locality of some sites. The correct historic collecting sites could be verified with the gazetteer of Mendes et al. (2013).

After Angola's independence in 1975 there was a long break in field research, with only a few records by various collectors. In January 2009, an expedition led by Brian Huntley visited the Serra da Chela in south-western Angola and the Namib Desert to the south: Warwick Tarboton recorded 47 species of Odonata around Humpata, of which five were recorded in Angola for the first time and one was new to science (Tarboton 2009, Dijkstra et al. 2015).

\section{Methods}

Jens Kipping surveyed the upper catchment of the Okavango (Cubango) River on the SAREP (Southern African Regional Environmental Program) Expedition from 5 to 22 May 2012 (18 field days). Specimens collected during a second SAREP expedition in April 2013 were also handed to Jens Kipping. Viola Clausnitzer and KD Dijkstra in collaboration with the Universidade Kimpa Vita (Uíge) and the Technical University of Dresden (Germany) surveyed around Uíge, Negage and N'dalantando in northern Angola in the wet season from 13 November to 1 December 2012 (19 days). Dijkstra revisited this area in the dry season, from 26 September to 5 October 2013 (10 days). Sara Fernandes Elizalde and Chris Hines provided photographic records in 2016. Adult dragonflies were usually caught with an aerial net and either identified in the field using Dijkstra and Clausnitzer (2014) or collected and identified later in the laboratory.

The authors also examined the Angolan collections and type material in the Natural History Museum in London, the National History Museum of Zimbabwe in Bulawayo 
(Dijkstra 2007a, b), the Royal Museum for Central Africa in Tervuren, Belgium and the Instituto de Investigação Agronómica in Huambo, Angola. All records are kept in the Odonata Database of Africa - ODA (Kipping et al. 2009) and mapped per species on African Dragonflies and Damselflies Online - ADDO (http://addo.adu.org.za/).

Specimens are currently at the Naturalis Biodiversity Center in Leiden, The Netherlands, including type material of eight species described by Dijkstra et al. (2015). Material must become available for study in the country of origin. Currently no facilities for permanent preservation and research of entomological specimens exist in Angola. As international scientific collaboration continues, natural history collections should be developed locally to receive and study this material.

The All Odonata Barcode Initiative at Naturalis Biodiversity Center generated sequences of the mitochondrial COI gene of the 4,260 specimens from sub-Saharan Africa, of which about 400 were from Angola. See Dijkstra et al. (2015) for details, including on DNA extraction, amplification (including primer combinations) and sequencing.

\section{Results}

The first SAREP Expedition yielded 87 species, 17 of them new to the country list and two new to science (Kipping 2012, Dijkstra et al. 2015). One additional species new for the country came from the second SAREP Expedition. The first expedition to Uíge, Negage and N'dalantando resulted in 138 species, of which 43 were recorded for the first time in Angola and five were new to science. The second visit produced 86 species, adding another 15 to the national list.

With the surveys in 2012 and 2013 and a careful review of the historic data, the known odonate fauna of Angola has increased from 158 species in the year 2011 to 234 species in 2013: an increase of about one-third with only 47 days in the field. Two species were added in 2016: Chris Hines photographed Tetrathemis camerunensis in Bengo Province and some male specimens of Bradinoypga strachani from N'dalantando were found in the collection of the Instituto de Investigação Agronómica in Huambo. ODA now holds 2,820 Angolan records from more than 250 localities.

The checklist of the Odonata of Angola is provided in Table 1, with notes on several species provided in the discussion. Table 2 lists 20 species that were removed from the country list. These were mentioned in one or more historic papers but represent likely misidentifications, taxonomic mix-ups, synonyms, etc. Table 3 lists species that should be present although reliable records are lacking. These are mostly found in border-rivers, especially on the well-studied Namibian bank of the Okavango (Suhling and Martens 2007). Many additional species occur in the well-surveyed Ikelenge Pedicle around the Zambezi source in north-western Zambia (Pinhey 1984, own surveys) immediately on the Angola border, but listing those species as likely to occur in the neighbouring Moxico Province of Angola would be speculative. 
Table I. Checklist of Odonata recorded from Angola.

\begin{tabular}{|c|c|c|c|}
\hline Species & English Name & $\mathbf{V}$ & $\mathbf{R L}$ \\
\hline \multicolumn{4}{|l|}{ Lestidae } \\
\hline Lestes amicus Martin, 1910 & Yellow-winged Spreadwing & 1 & LC \\
\hline Lestes dissimulans Fraser, 1955 & Cryptic Spreadwing & 1 & LC \\
\hline Lestes pallidus Rambur, 1842 & Pallid Spreadwing & 1 & LC \\
\hline Lestes pinheyi Fraser, 1955 & Pinhey's Spreadwing & 1 & LC \\
\hline Lestes plagiatus (Burmeister, 1839) & Highland Spreadwing & 1 & LC \\
\hline Lestes tridens McLachlan, 1895 & Spotted Spreadwing & 1 & LC \\
\hline Lestes virgatus (Burmeister, 1839) & Smoky Spreadwing & 3 & LC \\
\hline \multicolumn{4}{|l|}{ Calopterygidae } \\
\hline Phaon camerunensis Sjöstedt, 1900 & Emerald Demoiselle & $1 !$ & LC \\
\hline Phaon iridipennis (Burmeister, 1839) & Glistening Demoiselle & 1 & LC \\
\hline Sapho orichalcea McLachlan, 1869 \# & Mountain Bluewing & $1 !$ & LC \\
\hline Umma electa Longfield, 1933 & Metallic Sparklewing & 1 & LC \\
\hline Umma femina Longfield, 1947 & Angola Sparklewing & $1^{* *}$ & VU \\
\hline Umma longistigma (Selys, 1869) & Bare-bellied Sparklewing & 1 & $\mathrm{LC}$ \\
\hline Umma mesostigma (Selys, 1879) & Hairy-bellied Sparklewing & $1 !$ & LC \\
\hline \multicolumn{4}{|l|}{ Chlorocyphidae } \\
\hline Chlorocypha cancellata (Selys, 1879) & Exquisite Jewel & $1 !$ & LC \\
\hline Chlorocypha curta (Hagen in Selys, 1853) & Blue-tipped Jewel & $1 !$ & LC \\
\hline Chlorocypha cyanifrons (Selys, 1873) & Blue-fronted Jewel & $1 !$ & LC \\
\hline Chlorocypha fabamacula Pinhey, 1961 & Spotted Jewel & 1 & LC \\
\hline Chlorocypha victoriae (Förster, 1914) & Victoria’s Jewel & 1 & LC \\
\hline Platycypha angolensis Longfield, 1959 & Angola Dancing Jewel & $1^{* *}$ & NT \\
\hline Platycypha bamptoni (Pinhey, 1975) ${ }^{\#}$ & Highland Blue Jewel & $1^{* *}$ & $\mathrm{NE}$ \\
\hline Platycypha cf. bamptoni (Pinhey, 1975) \# & (near Highland Blue Jewel) & $1 !^{* * *}$ & $\mathrm{NE}$ \\
\hline Platycypha caligata (Selys, 1853) ${ }^{\#}$ & Common Dancing Jewel & 2 & LC \\
\hline Platycypha crocea (Longfield, 1947) ${ }^{\#}$ & Angola Blue Jewel & $1^{* *}$ & LC \\
\hline Platycypha rubriventris (Pinhey, 1975) ${ }^{\#}$ & Red-bellied Blue Jewel & $2^{* *}$ & DD \\
\hline Platycypha rufitibia (Pinhey, 1961) & Beautiful Jewel & 1 & LC \\
\hline \multicolumn{4}{|l|}{ Platycnemididae } \\
\hline Allocnemis nigripes (Selys, 1886) & Rainbow Yellowwing & 1 & LC \\
\hline Allocnemis pauli (Longfield, 1936) & Orange-tipped Yellowwing & $1 !$ & LC \\
\hline Copera congolensis (Martin, 1908) & Congo Featherleg & $1 !$ & LC \\
\hline Elattoneura acuta Kimmins, 1938 & Red Threadtail & $1 !$ & LC \\
\hline Elattoneura cellularis (Grünberg, 1902) ${ }^{\#}$ & Zambezi Threadtail & 3 & LC \\
\hline Elattoneura cf. glauca (Selys, 1860) \# & (near Common Threadtail) & 1 & LC \\
\hline Elattoneura lliba Legrand, 1985 & Eastern Stream Threadtail & $1 !$ & LC \\
\hline Elattoneura tarbotonorum Dijkstra, 2015 \# & Stout Threadtail & $2^{* *}$ & $\mathrm{DD}$ \\
\hline Mesocnemis cf. singularis Karsch, $1891^{\#}$ & (near Common Riverjack) & $1 !$ & $\mathrm{NE}$ \\
\hline \multicolumn{4}{|l|}{ Coenagrionidae } \\
\hline Aciagrion africanum Martin, 1908 & Blue Slim & 1 & LC \\
\hline Aciagrion macrootithenae Pinhey, 1972 & Awl-tipped Slim & 3 & DD \\
\hline Aciagrion nodosum (Pinhey, 1964) & Cryptic Slim & $1 !$ & LC \\
\hline Aciagrion rarum (Longfield, 1947) & Tiny Slim & 2 & $\mathrm{DD}$ \\
\hline Aciagrion steeleae Kimmins, 1955 & Swamp Slim & 3 & LC \\
\hline
\end{tabular}




\begin{tabular}{|c|c|c|c|}
\hline Species & English Name & V & RL \\
\hline Aciagrion zambiense Pinhey, 1972 & Zambia Slim & 3 & $\mathrm{DD}$ \\
\hline Africallagma fractum (Ris, 1921) & Southern Slender Bluet & 1 & LC \\
\hline Africallagma glaucum (Burmeister, 1839) & Swamp Bluet & 1 & LC \\
\hline Africallagma vaginale (Sjöstedt, 1917) & Forest Bluet & $1 !$ & LC \\
\hline Agriocnemis angolensis Longfield, 1947 & Blue Wisp & $1^{*}$ & LC \\
\hline Agriocnemis bumhilli Kipping, Suhling \& Martens, 2012 & Bumhill Wisp & $1 !^{*}$ & LC \\
\hline Agriocnemis canuango Dijkstra, 2015 & Bog Wisp & $1 !^{* *}$ & $\mathrm{DD}$ \\
\hline Agriocnemis exilis Selys, 1872 & Little Wisp & 1 & LC \\
\hline Agriocnemis forcipata Le Roi, 1915 & Greater Pincer-tailed Wisp & 1 & LC \\
\hline Agriocnemis cf. maclachlani Selys, $1877^{\text {\# }}$ & (near Forest Wisp) & $1 !$ & LC \\
\hline Agriocnemis ruberrima Balinsky, 1961 & Orange Wisp & $1 !$ & LC \\
\hline Agriocnemis toto Dijkstra, 2015 & Bruno's Wisp & $1 !^{* * *}$ & $\mathrm{DD}$ \\
\hline Agriocnemis victoria Fraser, 1928 & Lesser Pincer-tailed Wisp & 1 & LC \\
\hline Azuragrion nigridorsum (Selys, 1876) & Sailing Bluet & 1 & LC \\
\hline Ceriagrion annulatum Fraser, 1955 & Green-eyed Citril & $1 !$ & LC \\
\hline Ceriagrion bakeri Fraser, 1941 & Blue-fronted Citril & 3 & LC \\
\hline Ceriagrion corallinum Campion, 1914 & Green-fronted Citril & 1 & LC \\
\hline Ceriagrion glabrum (Burmeister, 1839) & Common Citril & 1 & LC \\
\hline Ceriagrion junceum Dijkstra \& Kipping, 2015 & Spikerush Citril & $1 !$ & LC \\
\hline Ceriagrion platystigma Fraser, 1941 & Variable Citril & 1 & LC \\
\hline Ceriagrion sakejii Pinhey, 1963 & Cream-sided Citril & $1 !$ & LC \\
\hline Ceriagrion suave Ris, 1921 & Plain Citril & 1 & LC \\
\hline Ceriagrion whellani Longfield, 1952 & Yellow-faced Citril & $1 !$ & LC \\
\hline Ischnura senegalensis (Rambur, 1842) & Tropical Bluetail & 1 & LC \\
\hline Pinheyagrion angolicum (Pinhey, 1966) & Pinhey's Bluet & 1 & LC \\
\hline Pseudagrion $(A)$ angolense Selys, 1876 & Angola Sprite & $1^{* *}$ & NT \\
\hline Pseudagrion (A) coeruleipunctum Pinhey, 1964 & Pretty Sprite & 3 & LC \\
\hline Pseudagrion $(A)$ estesi Pinhey, 1971 & Estes's Sprite & $1^{* *}$ & LC \\
\hline Pseudagrion (A) fisheri Pinhey, 1961 & Dark-tailed Sprite & 3 & LC \\
\hline Pseudagrion $(A)$ greeni Pinhey, 1961 & Clasper-tailed Sprite & 1 & LC \\
\hline Pseudagrion $(A)$ inconspicuum Ris, 1931 & Little Sprite & 1 & LC \\
\hline Pseudagrion $(A)$ kersteni Gerstäcker, 1869 & Powder-faced Sprite & 1 & LC \\
\hline Pseudagrion (A) kibalense Longfield, 1959 & Forest Sprite & 1 & LC \\
\hline Pseudagrion (A) makabusiense Pinhey, 1950 & Green-striped Sprite & 3 & LC \\
\hline Pseudagrion (A) melanicterum Selys, 1876 & Farmbush Sprite & 1 & LC \\
\hline Pseudagrion $(A)$ salisburyense Ris, 1921 & Slate Sprite & 1 & LC \\
\hline Pseudagrion $(A)$ sarepi Kipping \& Dijkstra, 2015 & Sarep Sprite & $1 !^{* *}$ & $\mathrm{DD}$ \\
\hline Pseudagrion $(A)$ serrulatum Karsch, 1894 & Superb Sprite & $1 !$ & LC \\
\hline Pseudagrion $(A)$ simonae Legrand, 1987 & Wide-striped Sprite & $1 !$ & LC \\
\hline Pseudagrion (B) acaciae Förster, 1906 & Acacia Sprite & 1 & LC \\
\hline Pseudagrion (B) coeleste Longfield, 1947 & Catshead Sprite & 1 & LC \\
\hline Pseudagrion $(B)$ deningi Pinhey, 1961 & Dark Sprite & $1 !$ & LC \\
\hline Pseudagrion $(B)$ dundoense Longfield, 1959 & Dundo Sprite & $2^{* *}$ & $\mathrm{DD}$ \\
\hline Pseudagrion (B) glaucescens Selys, 1876 & Blue-green Sprite & 1 & LC \\
\hline Pseudagrion (B) hamoni Fraser, 1955 & Swarthy Sprite & $1 !$ & LC \\
\hline Pseudagrion (B) helenae Balinsky, 1964 & Little Blue Sprite & $1 !$ & LC \\
\hline
\end{tabular}




\begin{tabular}{|c|c|c|c|}
\hline Species & English Name & $\mathbf{V}$ & $\mathbf{R L}$ \\
\hline Pseudagrion (B) isidromorai Compte Sart, 1967 & Large Blue Sprite & $1 !$ & LC \\
\hline Pseudagrion (B) massaicum Sjöstedt, 1909 & Masai Sprite & 1 & LC \\
\hline Pseudagrion (B) rufostigma Longfield, 1947 & Ruby Sprite & 1 & LC \\
\hline Pseudagrion $(B)$ sjoestedti Förster, 1906 & Variable Sprite & 1 & LC \\
\hline Pseudagrion (B) sublacteum (Karsch, 1893) & Cherry-eye Sprite & 1 & LC \\
\hline \multicolumn{4}{|l|}{ Aeshnidae } \\
\hline Afroaeschna scotias (Pinhey, 1952) & Shadow Hawker & $1 !$ & LC \\
\hline Anax congoliath Fraser, 1953 & Dark Emperor & $1 !$ & LC \\
\hline Anax ephippiger (Burmeister, 1839) & Vagrant Emperor & 1 & LC \\
\hline Anax imperator Leach, 1815 & Blue Emperor & 1 & LC \\
\hline Anax speratus Hagen, 1867 & Eastern Orange Emperor & 1 & LC \\
\hline Anax tristis Hagen, 1867 & Black Emperor & 1 & LC \\
\hline Gynacantha (A) sextans McLachlan, 1896 & Dark-rayed Duskhawker & 3 & LC \\
\hline Gynacantha (A) vesiculata Karsch, 1891 & Lesser Girdled Duskhawker & 3 & LC \\
\hline Gynacantha $(B)$ manderica Grünberg, 1902 & Little Duskhawker & 3 & LC \\
\hline Heliaeschna fuliginosa Karsch, 1893 & Black-banded Duskhawker & 1 & LC \\
\hline Heliaeschna ugandica McLachlan, 1896 & Uganda Duskhawker & 3 & LC \\
\hline Pinheyschna rileyi (Calvert, 1892) & Bullseye Hawker & 3 & LC \\
\hline \multicolumn{4}{|l|}{ Gomphidae } \\
\hline Crenigomphus cf. cornutus Pinhey, 1956" & (near Horned Talontail) & $1 !$ & LC \\
\hline Diastatomma selysi Schouteden, 1934 & Common Hoetail & 3 & LC \\
\hline Diastatomma soror Schouteden, 1934 & Painted Hoetail & 3 & LC \\
\hline Gomphidia quarrei (Schouteden, 1934) & Southern Fingertail & 3 & LC \\
\hline Ictinogomphus dundoensis Pinhey, 1961 & Swamp Tigertail & 1 & LC \\
\hline Ictinogomphus ferox (Rambur, 1842) & Common Tigertail & 1 & LC \\
\hline Ictinogomphus regisalberti (Schouteden, 1934) & Congo Tigertail & 3 & LC \\
\hline Lestinogomphus calcaratus Dijkstra, 2015 & Spurred Fairytail & $1 !$ & LC \\
\hline Libyogomphus tenaculatus Fraser, 1926 & Large Horntail & $1 !$ & LC \\
\hline Mastigogomphus chapini (Klots, 1944)\# & Western Snorkeltail & 2 & LC \\
\hline Microgomphus cf. nyassicus (Grünberg, 1902) \# & (near Eastern Scissortail) & $1 !$ & LC \\
\hline Neurogomphus alius Cammaerts, 2004 & Large Siphontail & $1 !$ & LC \\
\hline Notogomphus kimpavita Dijkstra \& Clausnitzer, 2015 & Angola Longleg & $1 !^{* * *}$ & $\mathrm{DD}$ \\
\hline Notogomphus praetorius (Selys, 1878) & Yellowjack Longleg & 2 & LC \\
\hline Notogomphus cf. spinosus (Karsch, 1890) \# & (near Jungle Longleg) & $1 !$ & LC \\
\hline Onychogomphus rossii Pinhey, 1966 & Angola Claspertail & $2^{* *}$ & $\mathrm{DD}$ \\
\hline Onychogomphus cf. styx Pinhey, $1961^{\#}$ & (near Northern Dark Claspertail) & $1 !$ & LC \\
\hline Paragomphus abnormis (Karsch, 1890) & Humdrum Hooktail & $1 !$ & LC \\
\hline $\begin{array}{l}\text { Paragomphus cf. darwalli Dijkstra, Mézière \& Papazian, } \\
2015^{\#}\end{array}$ & (near Darwall's Hooktail) & $1 !$ & $\mathrm{DD}$ \\
\hline Paragomphus genei (Selys, 1841) & Common Hooktail & 1 & LC \\
\hline Paragomphus machadoi Pinhey, 1961 & Forest Hooktail & 2 & LC \\
\hline Paragomphus cf. nigroviridis Cammaerts, 1969 \# & (near Black-and-green Hooktail) & $1 !$ & LC \\
\hline Phyllogomphus annulus Klots, 1944 & Crested Leaftail & 1 & LC \\
\hline Phyllogomphus selysi Schouteden, 1933 & Bold Leaftail & 3 & LC \\
\hline \multicolumn{4}{|l|}{ Macromiidae } \\
\hline Phyllomacromia aureozona (Pinhey, 1966) & Golden-banded Cruiser & $1 !$ & LC \\
\hline
\end{tabular}




\begin{tabular}{|c|c|c|c|}
\hline Species & English Name & V & RL \\
\hline Phyllomacromia contumax Selys, 1879 & Two-banded Cruiser & $1 !$ & LC \\
\hline Phyllomacromia hervei (Legrand, 1980) & River Cruiser & $1 !$ & LC \\
\hline Phyllomacromia melania (Selys, 1871) & Sombre Cruiser & 1 & LC \\
\hline Phyllomacromia overlaeti (Schouteden, 1934) & Clubbed Cruiser & 3 & LC \\
\hline Phyllomacromia paula (Karsch, 1892) & Greater Double-spined Cruiser & 3 & LC \\
\hline Phyllomacromia picta (Hagen in Selys, 1871) & Darting Cruiser & 3 & LC \\
\hline Phyllomacromia unifasciata (Fraser, 1954) & Golden-eyed Cruiser & 3 & LC \\
\hline \multicolumn{4}{|l|}{ Libellulidae } \\
\hline Acisoma inflatum Selys, 1882 & Stout Pintail & 1 & LC \\
\hline Acisoma trifidum Kirby, 1889 & Pied Pintail & 1 & LC \\
\hline Aethiothemis bequaerti Ris, 1919 & Skimmer-like Flasher & 1 & LC \\
\hline Aethiothemis ellioti (Lieftinck, 1969) & Plump Flasher & $1 !$ & LC \\
\hline Aethiothemis mediofasciata Ris, 1931 \# & Orange Flasher & 2 & LC \\
\hline Aethiothemis solitaria Martin, 1908 & Pearly Flasher & 1 & LC \\
\hline Aethriamanta rezia Kirby, 1889 & Pygmy Basker & 1 & LC \\
\hline Brachythemis lacustris (Kirby, 1889) & Red Groundling & 1 & LC \\
\hline Brachythemis leucosticta (Burmeister, 1839) & Southern Banded Groundling & 1 & LC \\
\hline Bradinopyga strachani (Kirby, 1900) & Red Rockdweller & 2 & LC \\
\hline Chalcostephia flavifrons Kirby, 1889 & Inspector & $1 !$ & LC \\
\hline Crocothemis brevistigma Pinhey, 1961 & Spotted Scarlet & $1 !$ & LC \\
\hline Crocothemis divisa Baumann, 1898 & Rock Scarlet & 1 & LC \\
\hline Crocothemis erythraea (Brullé, 1832) & Broad Scarlet & 1 & LC \\
\hline Crocothemis sanguinolenta (Burmeister, 1839) & Little Scarlet & 1 & LC \\
\hline Cyanothemis simpsoni Ris, 1915 & Bluebolt & $1 !$ & LC \\
\hline Diplacodes deminuta Lieftinck, 1969 & Little Percher & 1 & LC \\
\hline Diplacodes lefebvrii (Rambur, 1842) & Black Percher & 1 & LC \\
\hline Diplacodes luminans (Karsch, 1893) & Barbet Percher & 1 & LC \\
\hline Diplacodes pumila Dijkstra, 2006 & Dwarf Percher & $1 !$ & LC \\
\hline Eleuthemis eogaster Dijkstra, 2015 & Sunrise Firebelly & $1 !^{* *}$ & DD \\
\hline Eleuthemis libera Dijkstra \& Kipping, 2015 & Free Firebelly & $1 !$ & $\mathrm{DD}$ \\
\hline Hadrothemis camarensis (Kirby, 1889) & Saddled Jungleskimmer & 3 & LC \\
\hline Hadrothemis coacta (Karsch, 1891) & Robust Jungleskimmer & $1 !$ & LC \\
\hline Hadrothemis defecta (Karsch, 1891) & Scarlet Jungleskimmer & 3 & LC \\
\hline Hemistigma albipunctum (Rambur, 1842) & African Piedspot & 1 & LC \\
\hline Malgassophlebia bispina Fraser, 1958 & Ringed Leaftipper & $1 !$ & LC \\
\hline Micromacromia camerunica Karsch, 1890 & Stream Micmac & $1 !$ & LC \\
\hline Micromacromia flava (Longfield, 1947) & Angola Micmac & $1^{* *}$ & NT \\
\hline Neodythemis afra (Ris, 1909) & Seepage Junglewatcher & $1 !$ & LC \\
\hline Neodythemis klingi (Karsch, 1890) & Stream Junglewatcher & $1 !$ & LC \\
\hline Nesciothemis cf. farinosa (Förster, 1898) ${ }^{\#}$ & (near Eastern Blacktail) & 1 & LC \\
\hline Nesciothemis fitzgeraldi Longfield, 1955 & Lesser Peppertail & $1 !$ & LC \\
\hline Notiothemis robertsi Fraser, 1944 & Western Forestwatcher & $1 !$ & LC \\
\hline Olpogastra lugubris Karsch, 1895 & Bottletail & 1 & LC \\
\hline Orthetrum abbotti Calvert, 1892 & Little Skimmer & 1 & LC \\
\hline Orthetrum austeni (Kirby, 1900) & Giant Skimmer & 1 & LC \\
\hline Orthetrum brachiale (Palisot de Beauvois, 1817) & Banded Skimmer & 1 & LC \\
\hline
\end{tabular}




\begin{tabular}{|c|c|c|c|}
\hline Species & English Name & $\mathbf{V}$ & RL \\
\hline Orthetrum caffrum (Burmeister, 1839) & Two-striped Skimmer & 1 & LC \\
\hline Orthetrum chrysostigma (Burmeister, 1839) & Epaulet Skimmer & 1 & LC \\
\hline Orthetrum guineense Ris, 1910 & Guinea Skimmer & 1 & LC \\
\hline Orthetrum hintzi Schmidt, 1951 & Dark-shouldered Skimmer & 1 & LC \\
\hline Orthetrum icteromelas Ris, 1910 & Spectacled Skimmer & 1 & LC \\
\hline Orthetrum julia Kirby, 1900 & Julia Skimmer & 1 & LC \\
\hline Orthetrum machadoi Longfield, 1955 & Highland Skimmer & 1 & $\mathrm{LC}$ \\
\hline Orthetrum macrostigma Longfield, 1947 & Sharkfin Skimmer & 1 & $\mathrm{LC}$ \\
\hline Orthetrum microstigma Ris, 1911 & Farmbush Skimmer & 1 & LC \\
\hline Orthetrum monardi Schmidt, 1951 & Woodland Skimmer & 1 & LC \\
\hline Orthetrum robustum Balinsky, 1965 & Robust Skimmer & $1 !$ & LC \\
\hline Orthetrum saegeri Pinhey, 1966 & Mushroom Skimmer & $1 !$ & LC \\
\hline Orthetrum stemmale (Burmeister, 1839) & Bold Skimmer & 1 & LC \\
\hline Orthetrum trinacria (Selys, 1841) & Long Skimmer & 1 & LC \\
\hline Oxythemis phoenicosceles Ris, 1910 & Pepperpants & $1 !$ & LC \\
\hline Palpopleura albifrons Legrand, 1979 & Pale-faced Widow & $1 !$ & LC \\
\hline Palpopleura deceptor (Calvert, 1899) & Deceptive Widow & 3 & LC \\
\hline Palpopleura jucunda (Rambur, 1842) & Yellow-veined Widow & 1 & LC \\
\hline Palpopleura lucia (Drury, 1773) & Lucia Widow & 1 & LC \\
\hline Palpopleura portia (Drury, 1773) & Portia Widow & 1 & LC \\
\hline Pantala flavescens (Fabricius, 1798) & Wandering Glider & 1 & LC \\
\hline Porpax asperipes Karsch, 1896 & Powdered Pricklyleg & 1 & $\mathrm{LC}$ \\
\hline Porpax risi Pinhey, 1958 & Highland Pricklyleg & 1 & LC \\
\hline Rhyothemis fenestrina (Rambur, 1842) & Skylight Flutterer & 1 & LC \\
\hline Rhyothemis mariposa Ris, 1913 & Butterfly Flutterer & 2 & LC \\
\hline Rhyothemis semihyalina (Desjardins, 1832) & Phantom Flutterer & $1 !$ & LC \\
\hline Sympetrum fonscolombii (Selys, 1840) & Nomad & 2 & LC \\
\hline Tetrathemis camerunensis (Sjöstedt, 1900) & Forest Elf & 2 & LC \\
\hline Tetrathemis fraseri Legrand, 1977 & Treefall Elf & $1 !$ & LC \\
\hline Tetrathemis polleni (Selys, 1869) & Black-splashed Elf & 2 & LC \\
\hline Thermochoria equivocata Kirby, 1889 & Dash-winged Piedface & $1 !$ & LC \\
\hline Tholymis tillarga (Fabricius, 1798) & Twister & 1 & LC \\
\hline Tramea basilaris (Palisot de Beauvois, 1817) & Keyhole Glider & 1 & LC \\
\hline Trithemis aconita Lieftinck, 1969 & Halfshade Dropwing & $1 !$ & LC \\
\hline Trithemis annulata (Palisot de Beauvois, 1807) & Violet Dropwing & 1 & LC \\
\hline Trithemis anomala Pinhey, 1956 & Striped Dropwing & $1 !$ & LC \\
\hline Trithemis apicalis (Fraser, 1954) & Furtive Dropwing & $1 !$ & $\mathrm{LC}$ \\
\hline Trithemis arteriosa (Burmeister, 1839) & Red-veined Dropwing & 1 & LC \\
\hline Trithemis basitincta Ris, 1912 & Jungle Dropwing & $1 !$ & LC \\
\hline Trithemis dichroa Karsch, 1893 & Black Dropwing & 1 & LC \\
\hline Trithemis dorsalis (Rambur, 1842) & Highland Dropwing & 1 & LC \\
\hline Trithemis cf. dubia (Fraser, 1954) ${ }^{\#}$ & (near Sleek Dropwing) & $1 !$ & $\mathrm{DD}$ \\
\hline Trithemis furva Karsch, 1899 & Navy Dropwing & 1 & LC \\
\hline Trithemis imitata Pinhey, 1961 \# & Northern Fluttering Dropwing & $1 !$ & LC \\
\hline Trithemis integra Dijkstra, 2007 & Albertine Dropwing & $1 !$ & LC \\
\hline Trithemis kirbyi Selys, 1891 & Orange-winged Dropwing & 1 & LC \\
\hline
\end{tabular}




\begin{tabular}{|c|c|c|c|}
\hline Species & English Name & $\mathbf{V}$ & RL \\
\hline Trithemis leakeyi (Pinhey, 1956) & Mealy Dropwing & $1 !$ & LC \\
\hline Trithemis monardi Ris, $1931^{\#}$ & Southern Fluttering Dropwing & 1 & LC \\
\hline Trithemis nuptialis Karsch, 1894 & Hairy-legged Dropwing & 1 & LC \\
\hline Trithemis palustris Damm \& Hadrys, 2009 \# & Marsh Dropwing & $1 !$ & LC \\
\hline Trithemis pluvialis Förster, 1906 & Russet Dropwing & 1 & LC \\
\hline Trithemis pruinata Karsch, 1899 & Cobalt Dropwing & $1 !$ & $\mathrm{LC}$ \\
\hline Trithemis stictica (Burmeister, 1839) & Jaunty Dropwing & 1 & LC \\
\hline Trithemis werneri Ris, 1912 & Elegant Dropwing & 3 & LC \\
\hline Urothemis assignata (Selys, 1872) & Red Basker & 1 & LC \\
\hline Urothemis edwardsii (Selys, 1849) & Blue Basker & 1 & LC \\
\hline Zygonoides fuelleborni (Grünberg, 1902) & Southern Riverking & 3 & LC \\
\hline Zygonyx denticulatus Dijkstra \& Kipping, 2015 & Pale Cascader & $1 !$ & LC \\
\hline Zygonyx eusebia (Ris, 1912) & Imperial Cascader & 3 & LC \\
\hline Zygonyx flavicosta (Sjöstedt, 1900) & Ensign Cascader & 1 & LC \\
\hline Zygonyx natalensis (Martin, 1900) & Blue Cascader & 1 & LC \\
\hline Zygonyx regisalberti (Schouteden, 1934) & Regal Cascader & 1 & LC \\
\hline Zygonyx torridus (Kirby, 1889) & Ringed Cascader & 1 & LC \\
\hline
\end{tabular}

\# - see Taxonomic comments in the discussion

(V) Validation of species: "1!" new national record made by the authors; "1" records obtained by authors and confirming existing records; " 2 " specimens kept in collections (identification confirmed or primary types); " 3 " literature records, regarded as reliable because specimens were described well or location agrees with known biogeographic pattern; ${ }^{* *}$ - range restricted to Angola; ${ }^{*}$ - range restricted to Angola with very few exceptions (see Endemism in the discussion).

(RL) Global status according to the IUCN Red List of Threatened Species (2016): "CR" critically endangered, "DD" data-deficient, "EN" endangered, "NT" near-threatened, "VU" vulnerable, "LC" least concern, "NE" not evaluated.

Table 2. Odonata species that have been removed from the list for Angola.

\begin{tabular}{|c|c|c|}
\hline Species & $\begin{array}{c}\text { English } \\
\text { name }\end{array}$ & Reason for exclusion \\
\hline \multicolumn{3}{|l|}{ Platycnemididae } \\
\hline Elattoneura frenulata (Hagen in Selys, 1860) \# & $\begin{array}{c}\text { Sooty } \\
\text { Theadtail }\end{array}$ & See discussion. \\
\hline Mesocnemis singularis Karsch, 1891 \# & $\begin{array}{l}\text { Common } \\
\text { Riverjack }\end{array}$ & See discussion. \\
\hline \multicolumn{3}{|l|}{ Coenagrionidae } \\
\hline Aciagrion gracile (Sjöstedt, 1909) & $\begin{array}{l}\text { Graceful } \\
\text { Slim }\end{array}$ & $\begin{array}{l}\text { Pinhey (1972) reidentified a series collected at } \\
\text { Caianda (Pinhey 1964) as A.macrootithenae. }\end{array}$ \\
\hline Agriocnemis zerafica Le Roi, 1915 & Sahel Wisp & $\begin{array}{l}\text { Sahelian species unlikely to occur in Angola; } \\
\text { probably misidentification. }\end{array}$ \\
\hline Ceriagrion varians (Martin, 1908) & $\begin{array}{c}\text { Orange-red } \\
\text { Citril }\end{array}$ & $\begin{array}{l}\text { Taxonomic confusion with } C \text {. platystigma; } \\
\text { see Dijkstra et al. (2015). }\end{array}$ \\
\hline Pseudagrion (A) hageni Karsch, 1893 & $\begin{array}{l}\text { Painted } \\
\text { Sprite }\end{array}$ & $\begin{array}{l}\text { All Angolan records might refer to } P \text {. angolense } \\
\text { Selys, } 1876 \text { as that was formerly considered a } \\
\text { synonym of } P \text {. hageni, although the latter occurs in } \\
\text { DRC and Zambia close to the Angolan border. }\end{array}$ \\
\hline
\end{tabular}




\begin{tabular}{|c|c|c|}
\hline Species & $\begin{array}{c}\text { English } \\
\text { name }\end{array}$ & Reason for exclusion \\
\hline Pseudagrion $(A)$ spernatum Selys, 1881 & $\begin{array}{l}\text { Upland } \\
\text { Sprite }\end{array}$ & $\begin{array}{l}\text { Three records under the synonym Pseudagrion } \\
\text { gerstaeckeri Karsch, } 1899 \text { from highlands around } \\
\text { Huambo are far outside the known range. The } \\
\text { species might occur near the Zambian border. }\end{array}$ \\
\hline Pseudagrion $(A)$ monardi Longfield, 1947 & - & Synonym of $P$. angolense Selys, 1876. \\
\hline \multicolumn{3}{|l|}{ Gomphidae } \\
\hline Crenigomphus hartmanni (Förster, 1898) & $\begin{array}{l}\text { Clubbed } \\
\text { Talontail }\end{array}$ & $\begin{array}{l}\text { A female from Cubango (Longfield 1947) cannot } \\
\text { be reliably identified and falls outside the known } \\
\text { range. }\end{array}$ \\
\hline Gomphidia bredoi (Schouteden, 1934) & $\begin{array}{l}\text { Northern } \\
\text { Fingertail }\end{array}$ & $\begin{array}{l}\text { A female from Dundo (Pinhey 1961a) cannot } \\
\text { be reliably identified and falls outside the } \\
\text { known range. }\end{array}$ \\
\hline Microgomphus bivittatus Pinhey, 1961 & - & $\begin{array}{c}\text { Treated as a synonym to Mastigogomphus chapini } \\
\text { (see discussion). }\end{array}$ \\
\hline Paragomphus cognatus (Rambur, 1842) \# & $\begin{array}{c}\text { Rock } \\
\text { Hooktail }\end{array}$ & See discussion. \\
\hline Phyllogomphus dundomajoricus Fraser, 1957 & - & $\begin{array}{l}\text { Synonym of Phyllogomphus annulus } \\
\text { (Dijkstra et al. 2006). }\end{array}$ \\
\hline Phyllogomphus dundominusculus Fraser, 1957 & - & $\begin{array}{l}\text { Synonym of Phyllogomphus annulus } \\
\text { (Dijkstra et al. 2006). }\end{array}$ \\
\hline Phyllogomphus latifasciae Pinhey, 1961 & - & $\begin{array}{l}\text { Synonym of Phyllogomphus selysi } \\
\text { (Dijkstra et al. 2006). }\end{array}$ \\
\hline \multicolumn{3}{|l|}{ Macromiidae } \\
\hline Phyllomacromia aequatorialis Martin, 1906 & - & $\begin{array}{l}\text { A small series from Dundo (Pinhey 1961a) of } \\
\text { this poorly known species may have belonged } \\
\text { to another species such as P. hervei. }\end{array}$ \\
\hline \multicolumn{3}{|l|}{ Libellulidae } \\
\hline Acisoma panorpoides Rambur, 1842 & $\begin{array}{l}\text { Grizzled } \\
\text { Pintail }\end{array}$ & $\begin{array}{l}\text { Mens et al. (2016) showed that } A \text {. panorpoides is } \\
\text { an Asian species replaced in continental African } \\
\text { by two species of which } A \text {. inflatum has been } \\
\text { recorded in Angola. }\end{array}$ \\
\hline Eleuthemis buettikoferi Ris, 1910 & $\begin{array}{l}\text { Western } \\
\text { Firebelly }\end{array}$ & $\begin{array}{l}\text { Genus thought to be monotypic until new species } \\
\text { were described by Dijkstra et al. (2015), of which } \\
\text { two from Angola. E. buettikoferi is exclusively } \\
\text { West African. }\end{array}$ \\
\hline Trithemis donaldsoni (Calvert, 1899) & $\begin{array}{l}\text { Denim } \\
\text { Dropwing }\end{array}$ & $\begin{array}{l}\text { The record of two females from Dundo (Longfield } \\
\text { 1959) is doubtful as this sex is not easily identified } \\
\text { and the location falls outside the species' } \\
\text { known range. }\end{array}$ \\
\hline Zygonoides occidentis (Ris, 1912) & $\begin{array}{l}\text { Congo } \\
\text { Riverking }\end{array}$ & $\begin{array}{l}\text { Known from the Congo River basin. What } \\
\text { Longfield (1947) mentioned as Olpogastra } \\
\text { fuelleborni occidentis from Kakindo (Caquindo) is } \\
\text { most likely Zygonoides fuelleborni. }\end{array}$ \\
\hline Zygonyx speciosus Karsch, 1891 & $\begin{array}{l}\text { Specious } \\
\text { Cascader }\end{array}$ & $\begin{array}{l}\text { A rainforest species known mainly from } \\
\text { Cameroon and confused with } Z \text {. regisalberti. }\end{array}$ \\
\hline
\end{tabular}

for species marked with ${ }^{*}$ see Taxonomic comments in discussion 
Table 3. Odonata recorded from bordering rivers that most likely occur in Angola too.

\begin{tabular}{|c|c|c|}
\hline Species & English name & Nearest occurrence \\
\hline \multicolumn{3}{|l|}{ Platycnemididae } \\
\hline Mesocnemis singularis Karsch, 1891 & $\begin{array}{l}\text { Common } \\
\text { Riverjack }\end{array}$ & $\begin{array}{l}\text { Cunene, Okavango and Kwando Rivers } \\
\text { in northern Namibia. }\end{array}$ \\
\hline \multicolumn{3}{|l|}{ Coenagrionidae } \\
\hline Africallagma subtile (Ris, 1921) & Fragile Bluet & $\begin{array}{l}\text { Okavango and Oshikango Rivers } \\
\text { in northern Namibia. }\end{array}$ \\
\hline Agriocnemis gratiosa Gerstäcker, 1891 & $\begin{array}{l}\text { Gracious } \\
\text { Wisp }\end{array}$ & $\begin{array}{l}\text { Okavango and Kwando Rivers in } \\
\text { Namibian Caprivi Strip. }\end{array}$ \\
\hline Pseudagrion (A) spernatum Selys, 1881 & Upland Sprite & $\begin{array}{l}\text { At Jimbe and other rivers in Ikelenge Pedicle of } \\
\text { north-western Zambia. }\end{array}$ \\
\hline Pseudagrion (B) assegaii Pinhey, 1950 & Assegai Sprite & Kwando River in Namibian Caprivi Strip. \\
\hline Pseudagrion (B) sudanicum Le Roi, 1915 & $\begin{array}{l}\text { Blue-sided } \\
\text { Sprite }\end{array}$ & $\begin{array}{l}\text { Okavango and Kwando Rivers in } \\
\text { Namibian Caprivi Strip. }\end{array}$ \\
\hline \multicolumn{3}{|l|}{ Aeshnidae } \\
\hline Anax bangweuluensis Kimmins, 1955 & $\begin{array}{l}\text { Swamp } \\
\text { Emperor }\end{array}$ & $\begin{array}{l}\text { Common in the Okavango Delta (Kipping 2010) } \\
\text { and also known from the Okavango and Kwando } \\
\text { Rivers in the Namibian Caprivi. }\end{array}$ \\
\hline \multicolumn{3}{|l|}{ Gomphidae } \\
\hline $\begin{array}{l}\text { Crenigomphus kavangoensis Suhling } \\
\text { \& Marais, } 2010\end{array}$ & $\begin{array}{l}\text { Kavango } \\
\text { Talontail }\end{array}$ & Okavango River in Namibia. \\
\hline Lestinogomphus angustus Martin, 1911 & $\begin{array}{l}\text { Common } \\
\text { Fairytail }\end{array}$ & $\begin{array}{c}\text { Cunene, Okavango and Kwando Rivers in northern } \\
\text { Namibia. }\end{array}$ \\
\hline Lestinogomphus silkeae Kipping, 2010 & $\begin{array}{c}\text { Silke's } \\
\text { Fairytail }\end{array}$ & $\begin{array}{c}\text { One locality on the southern bank of the Okavango } \\
\text { River near Rundu, Namibia. }\end{array}$ \\
\hline $\begin{array}{l}\text { Mastigogomphus cf. dissimilis } \\
\text { (Cammaerts, 2004) }\end{array}$ & $\begin{array}{c}\text { (near } \\
\text { Southern } \\
\text { Snokeltail) } \\
\end{array}$ & $\begin{array}{l}\text { Larvae found at two places on the Namibian bank } \\
\text { of the Okavango (Suhling and Martens 2007). }\end{array}$ \\
\hline Paragomphus cataractae Pinhey, 1963 & $\begin{array}{l}\text { Cataract } \\
\text { Hooktail }\end{array}$ & $\begin{array}{l}\text { Waterfalls and rapids of the Cunene and } \\
\text { Okavango Rivers in northern Namibia. }\end{array}$ \\
\hline Neurogomphus cocytius Cammaerts, 2004 & $\begin{array}{l}\text { Kokytos } \\
\text { Siphontail }\end{array}$ & Okavango River in northern Namibia. \\
\hline Paragomphus elpidius (Ris, 1921) & $\begin{array}{l}\text { Corkscrew } \\
\text { Hooktail }\end{array}$ & $\begin{array}{l}\text { Cunene, Okavango and Kwando Rivers in northern } \\
\text { Namibia and the Ikelenge Pedicle of Zambia. }\end{array}$ \\
\hline Paragomphus sabicus Pinhey, 1950 & $\begin{array}{l}\text { Flapper } \\
\text { Hooktail }\end{array}$ & $\begin{array}{c}\text { Okavango and Kwando Rivers } \\
\text { in northern Namibia. } \\
\end{array}$ \\
\hline \multicolumn{3}{|l|}{ Libellulidae } \\
\hline Parazyxomma flavicans (Martin, 1908) & $\begin{array}{l}\text { Banded } \\
\text { Duskdarter }\end{array}$ & $\begin{array}{l}\text { Okavango and Kwando Rivers in } \\
\text { northern Namibia. }\end{array}$ \\
\hline Trithemis aequalis Lieftinck, 1969 & $\begin{array}{l}\text { Swamp } \\
\text { Dropwing }\end{array}$ & $\begin{array}{l}\text { Okavango and Kwando Rivers in } \\
\text { the Namibian Caprivi. }\end{array}$ \\
\hline Trithemis donaldsoni (Calvert, 1899) & $\begin{array}{c}\text { Denim } \\
\text { Dropwing }\end{array}$ & Okavango and Cunene Rivers in northern Namibia. \\
\hline Trithemis hecate Ris, 1912 & $\begin{array}{l}\text { Silhouette } \\
\text { Dropwing }\end{array}$ & $\begin{array}{l}\text { Common along the Cunene, Okavango and } \\
\text { Kwando Rivers in northern Namibia. }\end{array}$ \\
\hline $\begin{array}{l}\text { Trithemis morrisoni Damm \& Hadrys, } \\
2009\end{array}$ & $\begin{array}{c}\text { Rapids } \\
\text { Dropwing }\end{array}$ & $\begin{array}{l}\text { Okavango and Kwando Rivers in the Namibian } \\
\text { Caprivi (see note on T. palustris above). }\end{array}$ \\
\hline Trithetrum navasi (Lacroix, 1921) & Fiery Darter & $\begin{array}{l}\text { Cunene, Okavango and Kwando Rivers in } \\
\text { northern Namibia. }\end{array}$ \\
\hline
\end{tabular}




\section{Discussion}

\section{Taxonomic comments}

Sapho orichalcea - This species has been separated from S. gloriosa McLachlan, 1873 by the absence of a matt band on the otherwise wholly dark wings of both sexes (Pinhey 1971b). However, this band's extent and position is variable and the validity of these species in untested (Dijkstra unpub.). Angolan populations lack the band, consistent with $S$. orichalcea as currently defined.

Chlorocypha and Platycypha - Platycypha Fraser, 1949 is distinguished from Chlorocypha Fraser, 1928 only by the largely coloured and often widened male tibiae (Dijkstra and Clausnitzer 2014). However, COI sequences indicate that the blue Angolan species classified as Chlorocypha (bamptoni, crocea, rubriventris and a possible fourth species) and Platycypha angolensis (formerly a subspecies of P. caligata, see below) are closer to each other than to any Chlorocypha species, grouping nearer Platycypha (Dijkstra unpubl.). While P. angolensis (Fig. 6a) has legs typical of Plat$y c y p h a$, the blue Angola "Chlorocypha" males have black tibiae with white anterior faces, found also in many Chlorocypha species, but lack the yellow to red tibial markings found in other Platycypha. However, this area is paler than the femora and tarsi in females and fresh males of bamptoni and crocea. While the tibiae appear slender, those of the three species examined are actually expanded slightly and more than in Platycypha picta (Pinhey, 1962) and P. rufitibia. The black on the dorsum of the second abdominal segment (S2) shifted centrally onto the segment and covering its full extent also recalls typical Platycypha. Females have the black line on the entire dorsal abdominal carina present in all Platycypha but rare in Chlorocypha. The male of rubriventris has a red wash on the sides and underside of the thorax and abdomen, which is common in Platycypha but not found in Chlorocypha. Finally the resting behaviour with exposed tibiae as shown in Figure $5 \mathrm{~b}$ is also more typical of Platycypha than Chlorocypha (A. Günther in litt.). It thus seems warranted to transfer $P$. bamptoni, P. croced and P. rubriventris to Platycypha (new combinations). Separating Platycypha may make Chlorocypha paraphyletic, but the species transfer itself does not affect that.

Platycypha angolensis and P. caligata - While described as a subspecies of $P$. caligata, COI sequences of angolensis are nearer (but not identical) to those of crocea and cf. bamptoni than typical caligata from eastern and southern Afrca. Moreover, angolensis males have the thorax sides greenish with hardly a red tinge at maturity, the fore tibiae more abruptly widened at the base, the (especially hind) femora apically reddish and not contrasting with the tibiae, and black apical bars present on the abdominal segments up to S6 rather than S4 or S5. P. angolensis is thus treated as good species, which is widespread on and around the Angolan plateau. True $P$. caligata appears to extend into Angola from Zambia.

Platycypha bamptoni, Platycypha cf. bamptoni and P. crocea- The former was described as a subspecies of the latter but is easily separated in the field by its larger size, more 
robust build and distinct thoracic and abdominal markings. We propose to treat them as distinct species. Specimens like P. bamptoni from the upper Cubango differ from those of Tundavala and might represent an undescribed species.

Platycypha rubriventris - This species is only known from two males collected in 1965 at Teixeira de Sousa in north-eastern Angola. This should refer to the modern town of Luau on the DRC border, although its altitude (about 1,000 m) and location well to the east of the central plateau are surprising for an Angolan endemic.

Elattoneura cellularis, E. frenulata and E. tarbotonorum - Pinhey (1961b) reported a specimen from Lóvua near the Zambian border in eastern Angola as E. frenulata. The latter is confined to the Cape of South Africa, an error he corrected with the description of E. tropicalis Pinhey, 1974. That species, common in Zambia adjacent to Angola, was synonymised with E. cellularis by Dijkstra (2007b). Pinhey's (1975) record of E. frenulata from Serra da Chela, however, pertains to the recently described Angolan endemic E. tarbotonorum.

Elattoneura cf. glauca - Dijkstra et al. (2015) showed that E. glauca is genetically heterogeneous, specimens from Angola being most distinct and closer to specimens identified as E. cellularis from Katanga and Zambia. E. glauca males collected near Kinshasa, directly north of Angola, differ in penis morphology from typical East African males (Dijkstra unpubl.). As with other widespread species of southern and eastern Africa like Platycypha angolensis and Nesciothemis farinosa (see below), the geographically relatively isolated populations in the west may represent a distinct taxon.

Mesocnemis cf. singularis - Longfield (1947) and Pinhey (1965) reported a female of $M$. singularis from near Cubango in 1932. However, we doubt the identification because we found a species at localities across Angola that differs from typical $M$. singularis by the COI sequences and male appendages. Similar males are known from Gabon and Cameroon. Preliminary data suggest these and presumed $M$. singularis form a complex of three or more species (Dijkstra unpubl.).

Agriocnemis cf. maclachlani - Females in this genus are difficult to identify morphologically. The COI sequence of one collected near Uíge is nearest (but not identical to) that of males of $A$. maclachlani from central and western Africa.

Crenigomphus cf. cornutus - Females of this genus are difficult to identify. Those collected recently near N'dalantando and Cuchi are morphologically similar to females caught in copula with males of $C$. cornutus in Zambia close to the Angolan border. Mastigogomphus chapini - Pinhey (1961b) described Microgomphus bivittatus from a female from Dundo. Dijkstra (2007a) suggested that it might belong to Lestinogomphus, but Pinhey's drawings actually match the female of Mastigogomphus chapini and is considered synonymous (Dijkstra et al. 2015).

Microgomphus cf. nyassicus - Microgomphus identification is uncertain as long as the status of the potentially synonymous $M$. schoutedeni Fraser, 1949 is unresolved.

Notogomphus cf. spinosus - The larvae of this genus have not been treated taxonomically. The COI sequences of two collected near Uíge are nearest (but not identical to) those of six adults of $N$. spinosus from Gabon. 
Onychogomphus cf. styx - The taxonomy of the Afrotropical species placed in Onychogomphus is problematic, especially the dark species (Dijkstra 2007a). O. seydeli (Schouteden, 1934) from Zambia and Katanga and O. styx known mainly from Uganda cannot be separated reliably at present (Dijkstra and Clausnitzer 2014). Identification of Angolan specimens thus awaits revision of the group.

Paragomphus cognatus - Despite progress made by Dijkstra et al. (2015), the taxonomy of the cognatus-group of Paragomphus is problematic: especially older records of $P$. $\operatorname{cog}$ natus cannot be considered reliable, such as a female from Ebanga (Longfield 1945). Longfield (1959) stated that a male P. cognatus from Dundo differed from (topotypical) South African specimens by its markings, suggesting it belonged to another species. Pinhey (1961a) described $P$. machadoi from the same collection, but the holotype's collection dates differ from Longfield's (1959) male. Pinhey also mentioned $P$. alluaudi (Martin, 1915) from Dundo, but that is an eastern African species. Possibly he had the similar P. abnormis (Karsch, 1890) before him, which we recorded recently near Uíge. True $P$. cognatus is known from the Okavango and Cunene Rivers in northern Namibia and thus expected in southern Angola (Suhling and Martens 2007).

Paragomphus cf. darwalli and $P$. cf. nigroviridis - The larvae of this genus have not been treated taxonomically. The COI sequence of one larva collected near Uíge is nearest (but not identical to) that of ten adults described recently as $P$. darwalli from Gabon, while another from there is nearest (but not identical to) eight adults identified as P. nigroviridis from Liberia, Gabon and DRC (Dijkstra et al. 2015).

Aethiothemis mediofasciata - This species was described from a single female collected by Monard at Chimporo in southern Angola in 1928. While the holotype agrees with females associated with a species found from Liberia to Gabon and CongoBrazzaville, the recent rediscovery of A. gamblesi (Lieftinck, 1969) in Zambia casts doubt on its taxonomic status. Males are almost identical, the abdomen being orange with maturity in $A$. mediofasciata (as now known) and grey pruinose in $A$. gamblesi. No characters to separate females are known as yet. The Angolan type locality is highly isolated: $1,300 \mathrm{~km}$ south of the nearest recorded orange male and $900 \mathrm{~km}$ west of the nearest pruinose male. Likely only further records of males from Angola can resolve the issue.

Nesciothemis cf. farinosa - Large black-tipped Nesciothemis are currently classified as two species that differ mainly in the extent of pruinosity on the mature male abdomen: at most to the base of S4 in N. pujoli Pinhey, 1971 from western Africa, but up to the very base of $S 7$ in $N$. farinosa from southern and eastern Africa, although usually it does not extend beyond S5 and (more rarely) S6. However, in Angola and adjacent Namibia and Botswana mature males invariably have even the entire dorsum of S7 pruinose and sometimes the extreme base of S8 as well. COI sequences of Angolan specimens differ more from those of both $N$. pujoli and $N$. farinosa sampled across Africa, than these two differ from each other (Dijkstra unpubl.). Thus, an unnamed taxon may be present in south-western Africa.

Trithemis cf. dubia - Males from eastern Angola and Zambia differ in the shape of the hamule from those collected in Gabon, while the thoracic pattern differs from 
the female holotype of T. dubia from Lake Asebbe in Gabon. They belong to the longistyla-group of Trithemis with several species placed formerly in Anectothemis, Congothemis, Lokithemis and Porpacithemis (see Dijkstra et al. 2015).

Trithemis imitata and T. monardi - It is unclear whether these are distinct species or if

$T$. imitata is a paler and generally more northerly variety of $T$. monardi. Both are currently listed for Angola.

Trithemis palustris - Damm and Hadrys (2009) separated two species from T. stictica (Burmeister, 1839) on genetic and morphological grounds. However, they only provided genetic characters to distinguish $T$. palustris from its alleged sister species T. morrisoni Damm \& Hadrys, 2009. Our records may thus represent both taxa, but given the limited geographic scope of the 2009 study, these may also be synonymous.

\section{Composition}

Angola's rich dragonfly fauna expresses its geographic position, size and diversity (Fig. 2). Dijkstra et al. (2011) observed that roughly half of tropical African species occur predominantly within the extensive lowland forests of the western and central continent, a quarter is associated with the eastern and southern part dominated by highlands, while the remaining quarter occurs in open habitats throughout much of the Afrotropics. Indeed, about half of Angola's species are widespread across the continent and its exceptional diversity can be attributed to two major sources:

Almost 30\% are confined to forest habitats in the north, mostly below $1,000 \mathrm{~m}$ asl, e.g. Phaon camerunensis, Umma longistigma, Chlorocypha cancellata, Allocnemis pauli, Elattoneura lliba, Africallagma vaginale, Agriocnemis forcipata, Ceriagrion annulatum, Pseudagrion kibalense, Anax congoliath, Neurogomphus alius, Paragomphus machadoi, Phyllomacromia aureozona, Acisoma trifidum, Cyanothemis simpsoni, Hadrothemis camarensis, Malgassophlebia bispina, Micromacromia camerunica, Neodythemis klingi, Notiothemis robertsi, Orthetrum austeni, Oxythemis phoenicosceles, Porpax asperipes, Thermochoria equivocata, Trithemis basitincta and Zygonyx regisalberti. Nine species confined to the Lower Guinea, the forest area that stretches between the Congo Basin and Atlantic Ocean from Cameroon to Gabon and western Congo, reach their southern limit in north-western Angola: Sapho orichalcea, Chlorocypha cyanifrons, Platycypha rufitibia, Copera congolensis, Pseudagrion simonae, Paragomphus abnormis, P. cf. darwalli and Tetrathemis fraseri.

Nearly $20 \%$ favour the swamps, grasslands, miombo woodlands and gallery forests that stretch eastwards, mostly above 1,000 m asl. This fauna is concentrated in Katanga and northern Zambia, but has now been proven to extend across to the Angolan upland with species like Umma electa, Chlorocypha fabamacula, Aciagrion steeleae, Ceriagrion sakejii, Pinheyagrion angolicum, Pseudagrion greeni, P. inconspicuum, P. deningi, Phyllomacromia unifasciata, Aethiothemis ellioti, Crocothemis brevistigma, Eleuthemis libera, Nesciothemis fitzgeraldi, Orthetrum macrostigma, Porpax risi, Rhyothemis mariposa, 


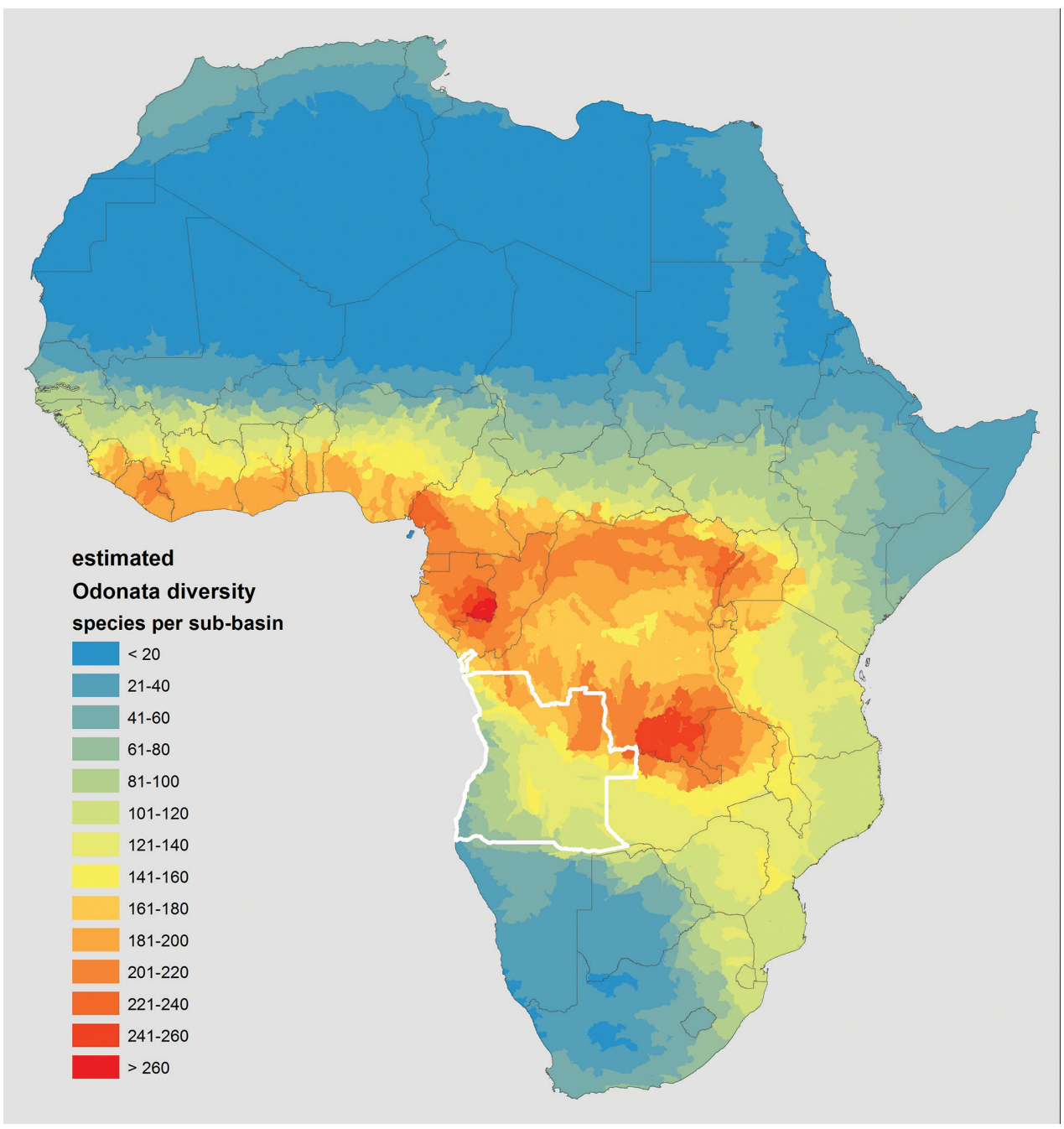

Figure 2. Distribution of Odonata diversity in continental Africa. Mapped as the number of species per Hydro1K basin (adapted from Clausnitzer et al. 2012), Angola outlined in white.

Trithemis anomala, T. leakeyi and Zygonyx denticulatus. The discovery of Trithemis integra near Uíge is of special interest, as it seemed endemic to the Albertine Rift, being known previously only from western Tanzania and Uganda and eastern DRC.

\section{Endemism}

Sixteen species have so far only been found in Angola. With the exception of two known only from their type locality, all are limited to the central plateau (Figs 3, 5): the type locality for Platycypha rubriventris is questionable (see comment above) as 


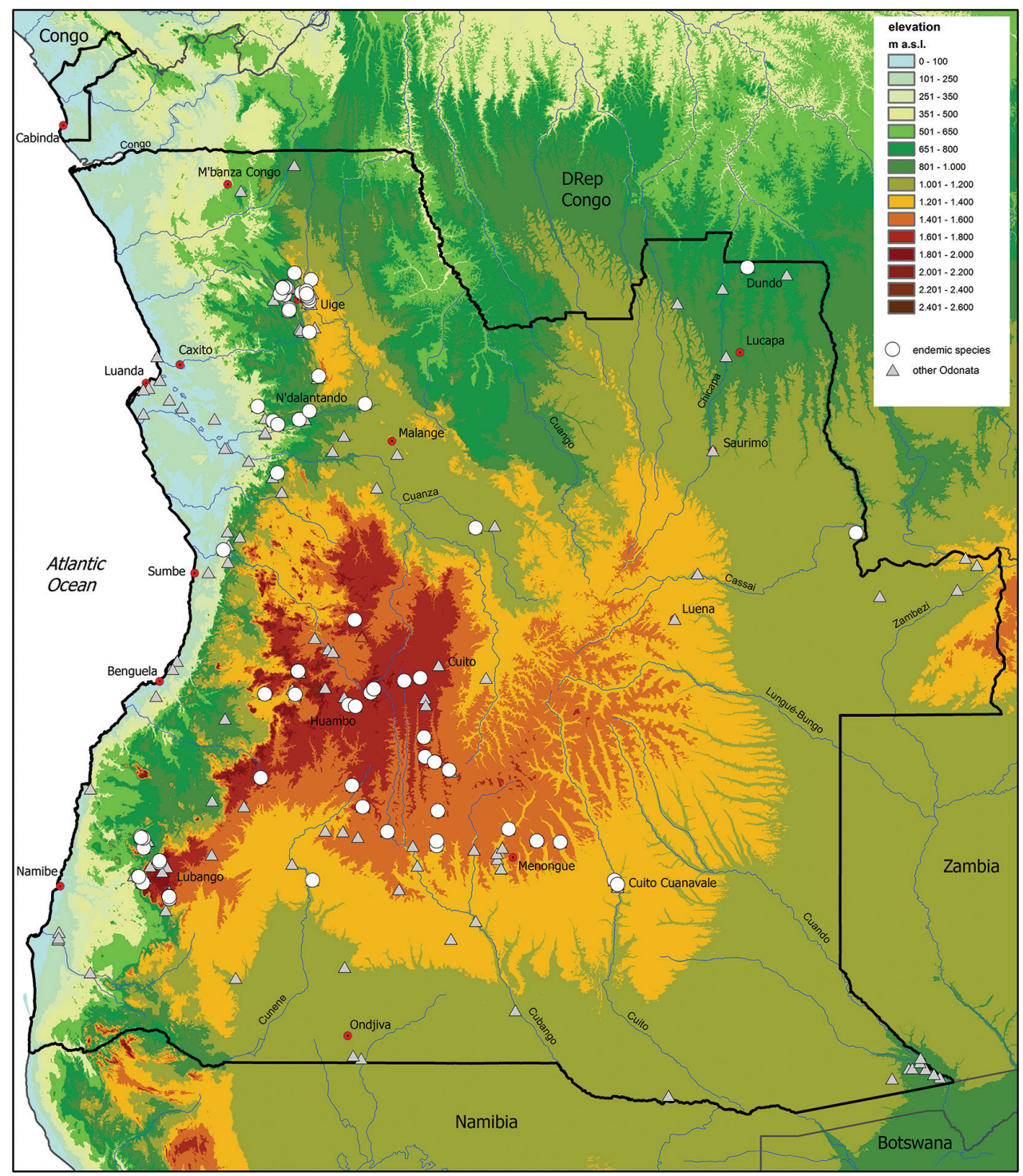

Figure 3. Records of 16 endemic Odonata species in Angola relative to altitude. White dots: endemic species (marked ${ }^{* *}$ in Table 1); grey triangles: other species. Elevation follows the GTOPO30 model of the U.S. Geological Center for Earth Resources Observation and Science (EROS).

may that of Pseudagrion dundoense from the extreme north-east, which could also be a river species from the very poorly sampled southern Congo Basin. No endemics have been found below 1,200 $\mathrm{m}$ asl in the east, although some drop down to about $500 \mathrm{~m}$ asl west of the escarpment. While the proportion of endemics $(7 \%)$ is lower than for Ethiopia (12 endemics; 11\%) and South Africa (30 endemics; 18\%), countries that also enclose distinct highland areas, this still ranks Angola as one of Africa's greatest 


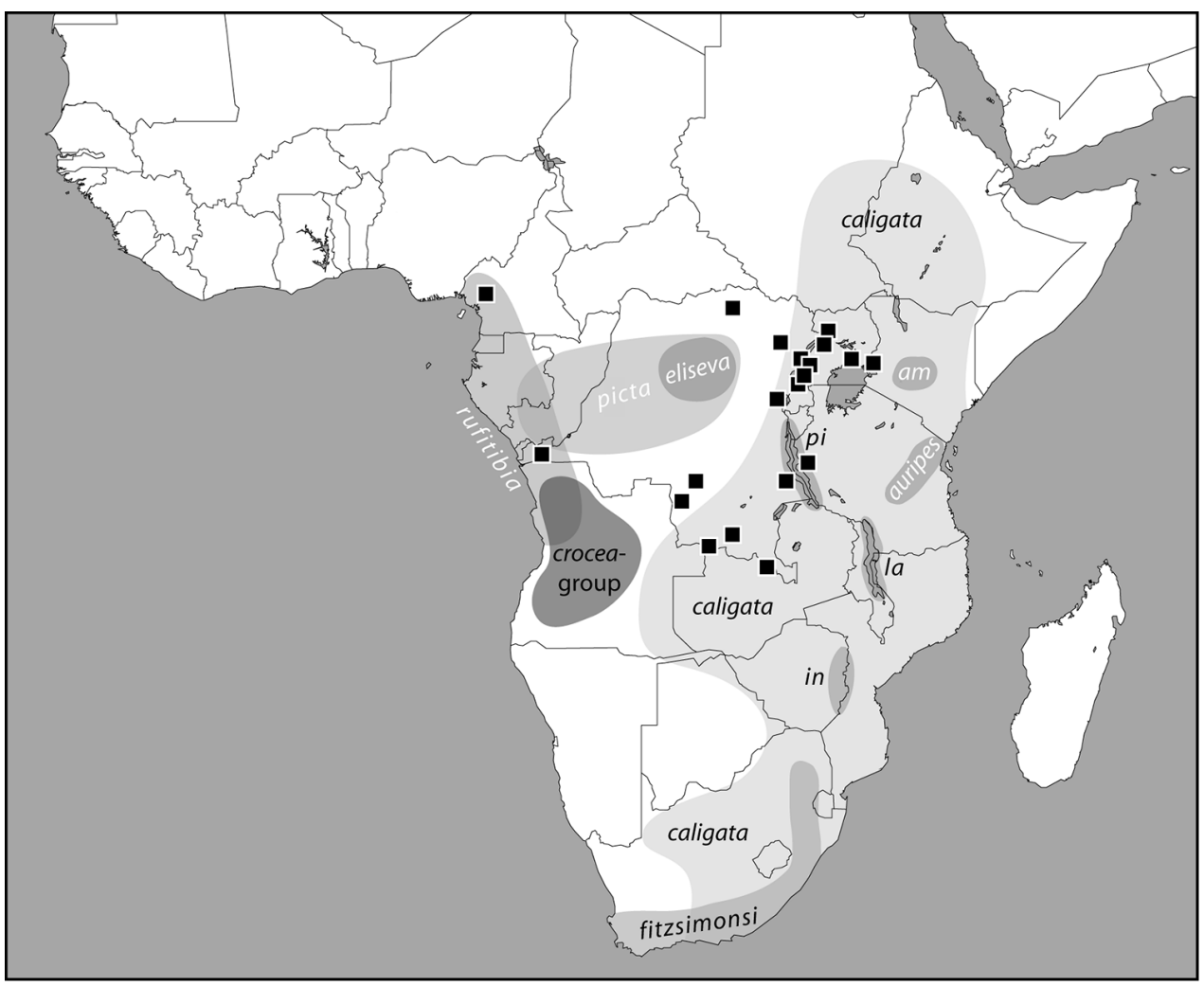

Figure 4. Distribution of the endemic crocea-group of Platycypha species and all congeneric taxa. Names of taxa that favour open habitats are in black, those tending toward forest in white (although P. rufitibia occurs on very open rivers); abbreviations: am $-P$. amboniensis, in $-P$. inyangae, la - form "lacus" of $P$. caligata which may represent a distinct species, pi $-P$. pinheyi. The rather dispersed forest species P. lacustris is shown with black squares (adapted from Dijkstra 2008).

centres of endemism for Odonata, rivalling the highlands of Cameroon (13 endemics) and the Albertine Rift, Eastern Arc and Katanga. Moreover, the number stands to increase, as almost two-fifths were described since exploration was reinitiated and at least another probable Platycypha species is already known to us.

Only Platycypha presents an endemic radiation. While the genus Chlorocypha has diversified with almost 30 species largely in the forested lowlands of west and central Africa, Platycypha is more varied and includes at least four open-land species (Fig. 4): P. caligata occurs widely from South Africa to Ethiopia, two species are limited to cooler parts of South Africa and Zimbabwe, one to Lake Tanganyika's shores, and another possibly to Lake Malawi. Two species of submontane forest are endemic to Tanzania and Kenya. Four species are mostly in forest, three in the Lower Guinea and Congo Basin, while P. lacustris stretches around that basin and is thus expected in Angola too. The Angolan endemics are found mainly between 1,300 and 1,800 m asl in open habitats. Such a local radiation of a group that has otherwise diversified in 

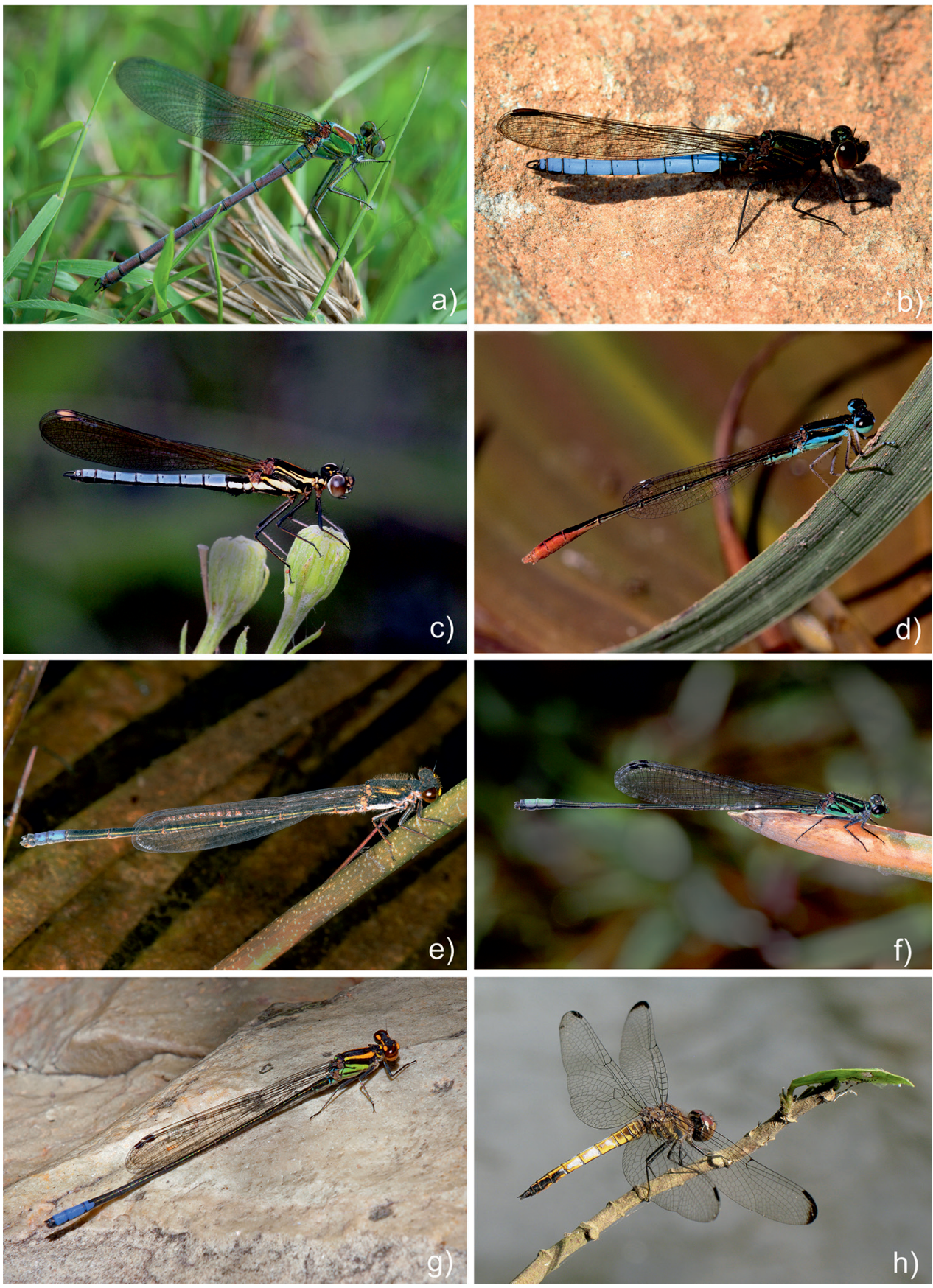

Figure 5. Photographs of some of Angola's (near) endemic dragonflies and damselflies. a Angola Sparklewing (Umma femina) b Highland Blue Jewel (Platycypha bamptoni) c Angola Blue Jewel (Platycypha crocea) d Blue Wisp (Agriocnemis angolensis) e Sarep Sprite (Pseudagrion sarepi) $\mathbf{f}$ Estes's Sprite (Pseudagrion estesi) $\mathbf{g}$ Angola Sprite (Pseudagrion angolense) $\mathbf{h}$ Sunrise Firebelly (Eleuthemis eogaster) (a S. Fernandes Elizalde b-f J. Kipping $\mathbf{g}$ W. Tarboton, h K.D. Dijkstra). 

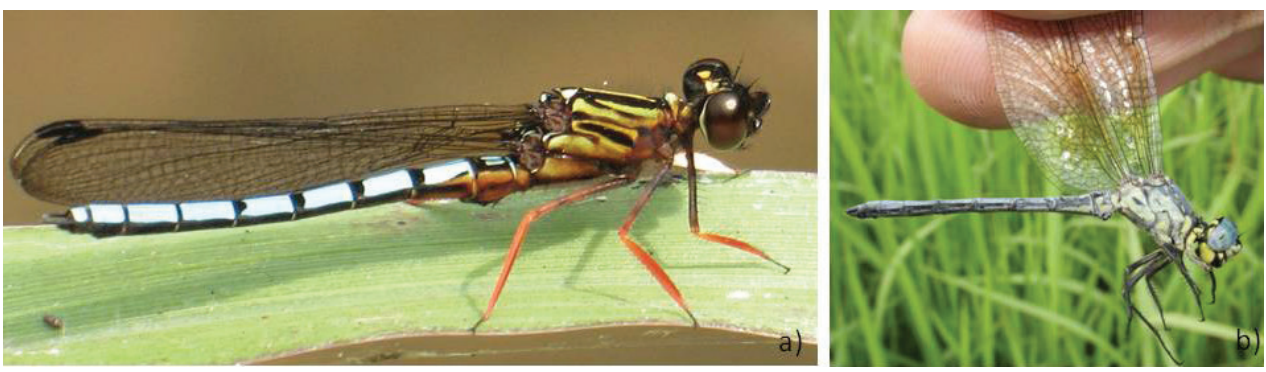

Figure 6. Photographs of Angola's endemic a Angola Dancing Jewel (Platycypha angolensis) and b Angola Micmac (Micromacromia flava) (K.D. Dijkstra).

the highlands to the east, and forests to the north, fits the overall affinities of Angola's endemic Odonata both geographically and ecologically.

The four endemic Pseudagrion species, for example, have separate origins but similar links: morphology and COI data suggest that the nearest relatives of $P$. angolense and $P$. estesi are the rainforest species $P$. grilloti Legrand, 1987 and $P$. kibalense respectively. The former is limited to Congo and Gabon but the latter extends to Cameroon and Uganda. $P$. sarepi is closely related to $P$. fisheri and $P$. greeni, both of which extend from Angola into Zambia. While these species belong to the genus's A-group, the B-group species $P$. dundoense is known only from Dundo and (as noted above) may not be endemic at all. Notogomphus kimpavita is the sister-species of $N$. praetorius found in highlands across southern Africa (including Angola), while Eleuthemis eogaster is nearest to an unnamed species from Gabon (Dijkstra et al. 2015). Molecular data for Umma femina and Onychogomphus rossii are lacking, but their morphology is close to U. electa and pale Onychogomphus species, both from the open plateaus stretching from Angola to Zambia and Katanga.

Thus, like the majority of Angola's Odonata, most endemics probably originated quite recently and proximally from the forests to the north and open habitats to the east. However, some affinities are unresolved and potentially more distant: Agriocnemis toto and especially $A$. canuango have no obvious close relatives (Dijkstra et al. 2015), while the nearendemic $A$. angolensis and $A$. bumbilli are probably related to each other but even more distinct overall (Dijkstra unpubl.). Indeed, Angola may be the centre of diversification of Africa's smallest damselflies. Micromacromia flava (Fig. 6b) is morphologically nearest $M$. miraculosa (Förster, 1906), known only from the East Usambara Mountains of north-eastern Tanzania (Dijkstra and Vick 2006), and the only one of four Micromacromia species adapted to non-forest habitats, being strongly pruinose with maturity. Elattoneura tarbotonorum may be closest to E. frenulata of south-western South Africa (Dijkstra et al. 2015).

\section{Potential for discovery}

If we compare the tallies for the well-studied neighbours Zambia and Namibia, the total number of species in Angola should lie somewhat above 300, meaning that less 
than $80 \%$ of the fauna is currently known. Additions can be expected throughout the country, but especially on the eastern and particularly northern border. Appropriately, the province of Lunda Norte should be the richest area of discovery, around Dundo where exploration began in the 1950s. On the other hand, the central highlands could expect more suprises, like the discovery of additional endemic species, with three areas being especially notable.

Firstly, despite having most records, the north-south directed range that lies entirely above 1,600 $\mathrm{m}$ asl and includes the Serra do Chilengue, Serra da Chela and Angola's highest peak at Mt Moco (2,620 m asl) is poorly sampled as the large gaps in Fig. 1 and 3 illustrate. Secondly, except for at its extreme northern and southern ends, the western escarpment has not been surveyed at all. Finally, a huge plateau at 1,200-1,600 m asl stretches east from the Bié Highlands. Except for its southern edge, this area shared between Bié and Moxico Provinces, which is almost as large as Uganda (or the United Kingdom), has no records. These deep Kalahari sands are the 'watertower' of Angola and its neighbours, incorporating the headwaters of the Cuito, Cuando, Chicapa, $\mathrm{Cu}$ ango, Cuanza and large tributaries of the Congo and Zambezi like the Cassai and Lungué-Bungo. The vast catchments of the Congo, Cuanza, Okavango and Zambezi meet in small area between Munhango and Cangonga. Watersheds are prone to endemism (Dijkstra et al. 2011) and this region is the top priority for further research.

New species are most likely to be found among genera prone to narrow (highland) ranges, i.e. with known Angolan endemics like Platycypha and Pseudagrion, but also Agriocnemis, Elattoneura, Notogomphus and perhaps Paragomphus. However, given the biogeographic diversity of Angola's fauna and endemics, we could expect greater surprises. Among forest genera with no known Angolan endemic, Allocnemis seems most likely to reveal one, e.g. on the escarpment. The presence (or local endemism) of distinctly Lower Guinean genera like Neurolestes, Africocypha, Pentaphlebia and Stenocnemis seems less likely, but the Lower Guinean Stenocypha gracilis (Karsch, 1899) has four endemic relatives in the Albertine Rift and the sister-taxon of the Upper and Lower Guinean Tragogomphus is Nepogomphoides stuhlmanni (Karsch, 1899) in the Eastern Arc, suggesting an Angolan taxon is possible.

Three typical African highland genera are notably absent from Angola. Atoconeura is most likely to be present, being found in Zambia, Katanga, the Lower Guinea and Albertine Rift. However, its absence also from South Africa suggests historical factors may have been limiting, e.g. that the highlands were uninhabitable in cooler periods and unreachable when habitats were suitable (Dijkstra 2006). This might not apply to Proischnura, present in South Africa as well as Cameroon and the Albertine Rift. However, that genus is absent from Katanga and northern Zambia, which lies lower and thus possibly provided no stepping-stone to the mountains of Angola. Similarly, Zosteraeschna extends from the Cape to the Albertine Rift, Ethiopia and even northern Namibia, but is absent from Katanga, northern Zambia and also Cameroon, potentially providing no route to Angola.

If these genera do occur in Angola, they might only occur above 1,400 $\mathrm{m}$ asl and could constitute endemic species. More probable, however, is the discovery of endem- 
ics in genera that are well-represented across the country and continent, and that have highland endemics elsewhere but not in Angola, such as Africallagma, Neodythemis and Orthetrum. The only record of Pinheyschna rileyi in upland Angola is of a female, which might also turn out to represent a distinctive population. Finally, there is a remote chance that a (mainly) South African genus like Syncordulia or Ceratogomphus is present. As the family Synlestidae is also represented by Nubiolestes in the Lower Guinea, while Chlorolestes is also probable.

\section{Conservation}

Our findings show that Angola's wealth of aquatic habitats harbours a rich freshwater fauna. Although large areas are relatively untouched, Angola's rapid economic and population growth will have a tremendous impact on the environment and thus human well-being in the future. In the light of this, Angola's development should consider (1) establishment of sewage works in cities and larger villages; (2) a stop on deforestation, especially along stream courses; (3) restoration of deforested water catchments; (4) village-level awareness campaigns for sustainable use of freshwater sources, e.g. no detergents and waste dumping in rivers; (5) biodiversity surveys and monitoring to feed into a national conservation plan.

Angola has agreed to the Aichi Targets of the Convention on Biological Diversity, including Target 12 that ensures that populations of threatened species do not decrease. Figure 3 shows where endemic species are found that should be taken into consideration for the National Biodiversity Strategy and Action Plan. However, their conservation status is uncertain at present. With the exception of four species, all endemics are currently considered Data Deficient for the IUCN Red List of Threatened Species. Platycypha angolensis, Pseudagrion angolense and Micromacromia flava are Near Threatened because, while they seem fairly widespread, their dependence on relatively natural habitats may put them at risk as human development progresses. Only Umma femina is now listed as threatened: known from only a few sites in the fairly densely populated highlands, it is thought to be Vulnerable to extinction. More research on all endemic species' statuses and ecology is required.

Angola has an exceptional fauna of dragonflies and damselflies, as well as many valuable rivers and wetlands. Odonata are excellent indicators of the health and biodiversity of both the freshwater and terrestrial realm. As the biological survey of Angola advances, they should be a priority taxon. Dragonflies are also positive symbols, as one soldier sent to Angola during the civil war wrote (Greg Bridges in litt.):

A memory that will never fade is watching dragonflies, in their variegated splendour of colour and dazzle, hovering and darting over stagnant pools. They helped me to transport to better things than war. If we found water to fill our canteens and I saw these little creatures, I would always try to get back to the pool later, by myself. And I would find a little piece of heaven. 


\section{Acknowledgements}

Jens Kipping is grateful to Dr. Paula Coelho (MINAMB) for making the survey in southern Angola possible, to Dr Chris Brooks of SAREP for the preparation and organization of the 2012 survey, to Martha Alexandre Zumbo (MINAMB), Maria Helena Loa (MINAMB), Julius Bravo (MINAMB), Francisco de Almeida (INIP), Manuel Domingos (INIP) and Gabriel Cabinda (Agriculture and Rural Development and Fisheries) for their help in organization and management on the tour, and to Vince Shacks and Werner Conradie for collecting specimens on the second SAREP survey. Viola Clausnitzer and KD Dijkstra thank Alvaro Bruno Toto Nienguesso, the driving force behind biodiversity research in Uíge Province, Angola, Prof Dr Neinhuis and Dr Thea Lautenschlaeger from TU Dresden for inviting us to join the field survey in the Uíge province. The fieldwork in Angola was supported by a travel fund from the German Academic Exchange Service (DAAD). These published results were obtained in collaboration with the Instituto Nacional da Biodiversidade e Áreas de Conservação (INBAC) of the Ministério do Ambiente da República de Angola. Chris Hines provided photographic records and Warwick Tarboton gave permission to use his records and photograph.

\section{References}

Clausnitzer V, Koch R, Dijkstra K-DB, Boudot J-P, Kipping J, Samraoui B, Samways MJ, Simaika JP, Suhling F (2012) Focus on African freshwaters: hotspots of dragonfly diversity and conservation concern. Frontiers in Ecology and the Environment 10: 129-134. https://doi.org/10.1890/110247

Damm S, Hadrys H (2009) Trithemis morrisoni sp. nov. and Trithemis palustris sp. nov. from the Okavango and Upper Zambezi Floodplains previously hidden under T. stictica (Odonata: Libellulidae). International Journal of Odonatology 12(1): 131-145. https://doi.org /10.1080/13887890.2009.9748333

Dijkstra, K-DB (2006) The Atoconeura problem revisited: taxonomy, phylogeny and biogeography of a dragonfly genus in the highlands of Africa (Odonata, Libellulidae). Tijdschrift voor Entomologie 149: 121-144. https://doi.org/10.1163/22119434-900000193

Dijkstra K-DB (2007a) The name-bearing types of Odonata held in the Natural History Museum of Zimbabwe, with systematic notes on Afrotropical taxa. Part 1: introduction and Anisoptera. International Journal of Odonatology 10(1): 1-29. https://doi.org/10.1080/1 3887890.2007.9748285

Dijkstra K-DB (2007b) The name-bearing types of Odonata held in the Natural History Museum of Zimbabwe, with systematic notes on Afrotropical taxa. Part 2: Zygoptera and description of new species. International Journal of Odonatology 10(2): 137-170. https:// doi.org/10.1080/13887890.2007.9748296

Dijkstra K-DB (2008) The Systematist's Muse - two new damselfly species from 'Elisabetha' in the Congo Basin (Odonata: Chlorocyphidae, Platycnemididae). Zoologische Mededelingen Leiden 82: 15-27. 
Dijkstra K-DB, Vick GS (2006) Inflation by venation and the bankruptcy of traditional genera: the case of Neodythemis and Micromacromia, with keys to the continental African species and the description of two new Neodythemis species from the Albertine Rift (Odonata: Libellulidae). International Journal of Odonatology 9: 51-70. https://doi.org/10.10 80/13887890.2006.9748263

Dijkstra K-DB, Clausnitzer V, Vick GS (2006) Revision of the three-striped species of Phyllogomphus (Odonata, Gomphidae). Tijdschrift voor Entomologie 149: 1-14. https://doi. org/10.1163/22119434-900000183

Dijkstra K-DB, Boudot J-P, Clausnitzer V, Kipping J, Kisakye JJ, Ogbogu SS, Samraoui B, Samways MJ, Schütte K, Simaika JP, Suhling F, Tchibozo SL (2011) Chapter 5. Dragonflies and damselflies of Africa (Odonata): history, diversity, distribution, and conservation. In: Darwall WRT, Smith KG, Allen DJ, Holland RA, Harrison IJ, Brooks EGE (Eds) The Diversity of Life in African Freshwaters: Under Water, Under Threat. An analysis of the status and distribution of freshwater species throughout mainland Africa. IUCN, Cambridge and Gland, $347 \mathrm{pp}$.

Dijkstra K-DB, Clausnitzer V (2014) The Dragonflies and Damselflies of Eastern Africa: Handbook for all Odonata from Sudan to Zimbabwe. Studies in Afrotropical Zoology 298. Tervuren: Royal Museums for Central Africa, 263 pp.

Dijkstra K-DB, Kipping J, Mézière N (2015) Sixty new dragonfly and damselfly species from Africa (Odonata). Odonatologica 44(4): 447-678.

Kipping J, Dijkstra K-DB, Clausnitzer V, Suhling F, Schütte K (2009) Odonata Database of Africa (ODA). Agrion 13: 20-23.

Kipping J (2010) The dragonflies and damselflies of Botswana - an annotated checklist with notes on distribution, phenology, habitats and Red List status of the species (Insecta: Odonata). Mauritiana (Altenburg) 21: 126-204.

Kipping J (2012) Southern African Regional Environmental Program (SAREP) - First Biodiversity Field Survey Upper Cubango (Okavango) catchment, Angola, May 2012 - Dragonflies \& Damselflies (Insecta: Odonata), Expert Report 2012: 1-108.

Longfield C (1936) Studies on African Odonata, with synonymy and descriptions of new species and subspecies. Transactions of the Royal Entomological Society of London 85: 467-499. https://doi.org/10.1111/j.1365-2311.1936.tb00240.x

Longfield C (1947) The Odonata of South Angola: Results of the Mission Scientifiques Suisses 1928-29, 1932-33. Arquivos do Museu Bocage 16: 1-31.

Longfield C (1955) The Odonata of North Angola, part 1. Publicaçóes Culturais Companhia de Diamantes de Angola 27: 11-64.

Longfield C (1959) The Odonata of North Angola, part 2. Publicaçóes Culturais Companhia de Diamantes de Angola 45: 16-42.

Mendes LF, Bivar de Sousa A, Figueira R, Serrano ARM (2013) Gazetteer of the Angolan localities known for beetles (Coleoptera) and butterflies (Lepidoptera: Papilionoidea). Boletim da Sociedade Portuguesa de Entomologia 228(VIII-14): 257-292.

Mens L, Schütte K, Stokvis FR, Dijkstra K-DB (2016) Six, not two, species of Acisoma pintail dragonfly (Odonata: Libellulidae). Zootaxa 4109(2): 153-172. https://doi.org/10.11646/ zootaxa.4109.2.3 
Pinhey ECG (1961a) A collection of Odonata from Dundo, Angola with the descriptions of two new species of Gomphids. Publicações Culturais Companhia de Diamantes de Angola 56: 71-78.

Pinhey ECG (1961b) Some dragonflies (Odonata) from Angola and descriptions of three new species of the family Gomphidae. Publicaçôes Culturais Companhia de Diamantes de Angola 56: 79-86.

Pinhey ECG (1964) Dragonflies (Odonata) of the Angola-Congo borders of Rhodesia. Publicaçôes Culturais Companhia de Diamantes de Angola 63: 97-130.

Pinhey ECG (1965) Odonata from Luanda and the Lucala River, Angola. Revista de Biologia 5: 159-164.

Pinhey ECG (1966) New distributional records for African Odonata and notes on a few larvae. Arnoldia Rhodesia 2(26): 1-5.

Pinhey ECG (1971a) Notes on the genus Pseudagrion Selys (Odonata: Coenagrionidae). Arnoldia Rhodesia 5(6): 1-4.

Pinhey ECG (1971b) Odonata collected in Republique Centre-Africaine by R. Pujol. Arnoldia Rhodesia 5(18): 1-16.

Pinhey ECG (1974) A revision of the African Agriocnemis Selys and Mortonagrion Fraser (Odonata: Coenagrionidae). Occasional Papers of the National Monuments of Rhodesia B 5/ 4: 171-278.

Pinhey ECG (1975) A collection of Odonata from Angola. Arnoldia Rhodesia 7(23): 1-16.

Pinhey ECG (1984) A check-list of the Odonata of Zimbabwe and Zambia. Smithersia 3: $1-64$.

Ris F (1931) Odonata aus Süd-Angola. Revue Suisse Zoologie 38(7): 97-112.

Suhling F, Martens A (2007) Dragonflies and damselflies of Namibia. Gamsberg Macmillan, Windhoek, $280 \mathrm{pp}$.

Tarboton W (2009) SANBI/ISCED/ANU Expedition to Southern Angola, January 2009: A dragonfly survey of the Humpata District. Draft Report, 1-3.

Vick GS, Chelmick DG, Martens A (2001) In memory of Elliot Charles Gordon Pinhey (10 July 1910-7 May 1999). Odonatologica 30: 1-11. 